\title{
APLICACÃO DE MODELOS LINEARES EM EXPERIMENTOS COM DADOS CATEGORIZADOS
}

\author{
DALTON GERALDO GUAGLIANONI \\ LICENCIADO EM MATEMATICA
}

Orientadora: Profạ Drạ MARLI DE BEM GOMES

Dissertação apresentada à Escola Superior de Agricultura "Luiz de Queiroz" da Universidade de São Paulo para obtenção do título de Mestre em Agronomia. Ârea de Con centração: "Estatística e Experi mentação Agronômica".

PIRACICABA

Estado de São Paulo - Brasil

Outubro de 1986 
ii.

$\bar{\AA}$

Neusa Maria Nunes Guaglianoni

Patrick Nunes de Oliveira Guaglianoni

Erick Nunes de Oliveira Guaglianoni

Elvira Borelli Guaglianoni 


\section{AGRADECIMENTOS}

A Professora Doutora Marly de Bem Gomes pela orientação, sugestões, compreensão e amizade.

Ao Professor Ròmeu Magnani pelo companheirismo, incentivo e amizade demonstrados em todas as horas.

Aos Docentes do Departamento de Matemática e Estatística ' da ESALQ/USP pelos bons ensinamentos transmitidos.

A Maria Izalina Ferreira Alves, Octávio Frassetto, Rosa Maria Alves pelas gentilezas e prestatividades.

Ao Professor Doutor Carlos Felício Vanni e Professora Doutora Maria Aparecida Rodrigues de Lima Grande Chefes do Departamen to de Ciências da Educação do ILCSE/UNESP pelo incentivo e compreensão os quais concorreram para a realização deste trabalho.

Aos amigos José Sebastião Soares e Luís Fernando Coletti pelos serviços de datilografia prestados. 
PĀGINA

RESUMO

3.2. Tabela de Classificação Cruzadas mais Usuais

3.3. Aplicação do Mētodo "GSK" em um Experimento

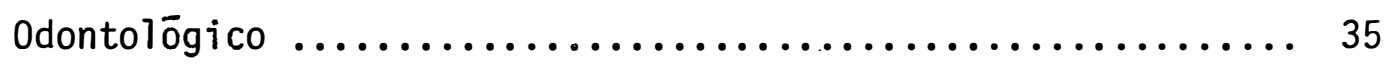

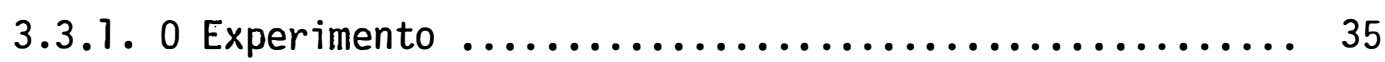

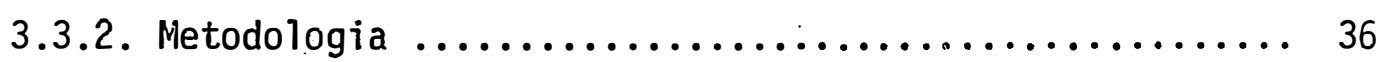

3.3.3. Anālise do experimento pelomodelo GSK ........... 39

3.3:3.1. Matriz das proporções observadas $\mathrm{p} \ldots \ldots \ldots \ldots \ldots \ldots \ldots \ldots \ldots \ldots \ldots . \ldots \ldots$

3:3.3.2. Matriz de variāncias e covariāncias $\underset{\sim}{V}(p) \ldots \ldots \ldots \ldots \ldots \ldots \ldots \ldots \ldots \ldots$

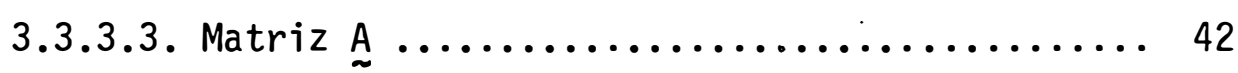

3.3.3.4. Matriz $x \ldots \ldots \ldots \ldots \ldots \ldots \ldots \ldots \ldots \ldots \ldots \ldots$

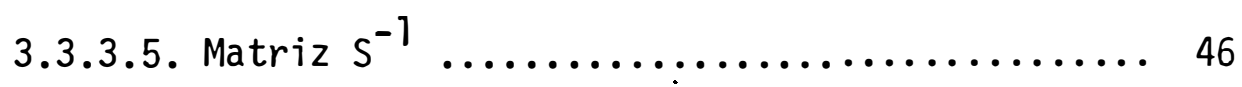

3.3.3.6. Matriz $F(p) \ldots \ldots \ldots \ldots \ldots \ldots \ldots \ldots \ldots$

3.3.3.7. Matriz $\beta \ldots \ldots \ldots \ldots \ldots \ldots \ldots \ldots \ldots \ldots$

3.3.3.8. A estimativa do modelo ............. 49

3.3.3.9. Teste dos parämetros Encontrados 
3.3.4. Readaptando o modelo .................... 52

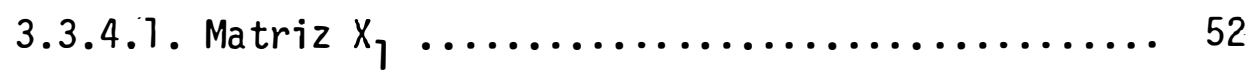

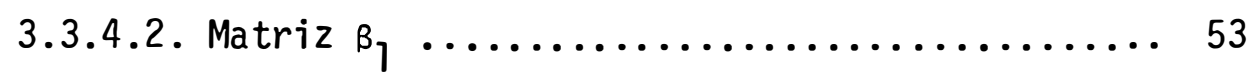

3.3.4.3. Estimando o novo modelo

3.3.4.4. As proporções obtidas e as estimadas atravēs do modelo reduzido ........................... 53

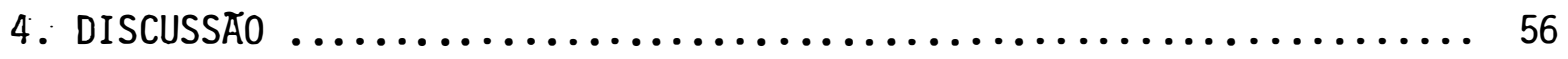

5. CONCLUSOES FINAIS ............................ 61

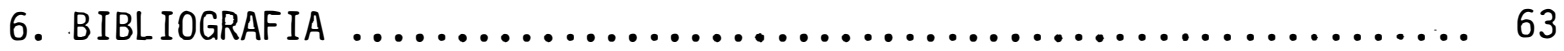

7. BIBL IOGRAFIA COMPLEMENTAR $\ldots \ldots \ldots \ldots \ldots \ldots \ldots \ldots \ldots \ldots \ldots \ldots \ldots \ldots \ldots \ldots \ldots \ldots$

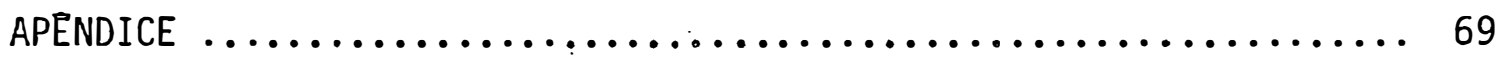




\section{APLICAÇAOO DE MODELOS LINEARES EM \\ EXPERIMENTOS COM DADOS CATEGORIZADOS}

Autor: 'DALTON GERALDO GUAGLIANONI

Orientadora: Marli de Bem Gomes

RESUMO

Pesquisas de diversas áreas como: Biologia, Me dicina, Psicologia, Sociologia, Agricultura etc, apresentam como resultados dados qualitativos o que nos leva a, distribuí-los em algumas categorias de respostas, razão principal da denominação de dados categorizados. Nas ūitimas décadas, da da uma intensa utilização, esse tipo de dados passou a ser ob jeto de estudo de diversos pesquisadores no intuito de tornar mais flexível as hipóteses a serem testadas, uma vez que as soluções usuais não permitem nenhuma flexibilidade.

0 objetivo geral do presente trabalho referese ao estudo do método proposto por GRIZZLE et alli no qual propõe uma teoria utilizando modelos de regressão 1inear para solucionar problemas envolvendo tabelas de contingência.

E objetivo fundamental deste trabalho aplicar 
vii.

o método em um experimento odontológico onde os dados são classificados em cinco fatores subdivididos em dois níveis ca da um, e mostrar que as matrizes envolvidas são de fácil cons trução, após tal construção, o método pode ser processado por um microcomputador através de um programa relativamente simples envolvendó produtos de inversão de matrizes.

Pudemos com a teoria de GRIZZLE et alli ajus tar uma superfície de resposta ao experimento odontológico com bons resultados e concluir ao final que o método é dè fácil aplicação e possibilita uma grande diversificação de hipó teses a serem testadas. 
LINEAR MODELS APPLICATION TO

CATEGORICAL DATA EXPERIMENTS

Author: $\quad$ DALTON GERALDO GUAGLIANONI

Adviser: Marli de Bem Gomes

SUMMARY

Researches of several areas like: Biology, Medicine, Psychology, Sociology, Agriculture, etc, present like results qualitative data that conducts us to distribute them in some categories of answers, principal reason by denomination of categorical data.

In the last decades, because of a intensive utilization, this type of points passed to be the object of estudy of several searchers in the intent to turn more flexible the hypothesis to be examined, considering that usual solutions do not permit any flexibility.

General objective of the present work refers to the study of the method proposed by GRIZZLE. et alii (1969) in which proposes a theory utilizing models of linear 
regression to solve problems involving tables of contingency. It is fundamental objective of this work to apply the method in an odontological experiment where the elements are classified in five factors subdivided in two involved matrixes are of easy construction, and following this the method may be prosecuted by a micron computer through a program more or less simple involving products of inversion of matrixes .

With the theory by Grizzle, Starmer and Koch we could adjust a response surface to the odontological experiment with good results and we could conclude at the final that the method is of easy application and it makes possible a great diversification of hypothesis to be tested. 
1. INTRODUÇÃO

As pesquisas nas āreas de biologia, medicina, psicologia e sociologia, entre outras, apresentam frequentemente seu resultado expresso em dados qualitativos, o que nos leva a distribuí-los em algumas categorias de réspostas, razão principal da denominação de dados categorizados, normalmente apresentados em tabelas de contingências. Esses dados são frequências com que indivíduos, objetos, etc., são class ficados através das diversas categorias de resposta relativas ao experimento. Essas frequências seguem uma distribuição mul tinomial.

As hipóteses relativàs a dados catégorizados são normalmente testadas através da estatística $x^{2}$ de Pearson, $x_{1}^{2}$ modificado de Neyman e do teste da razão de Verossimilhança. 
Devido ao crescente uso de pesquisas com tais características e a necessidade de maior flexibilidade nas hí póteses a serem testadas, o assunto passou a ser objeto de es tudo de muitos pesquisadores.

GRIZZLE et alii (1969) propuseram-se a utilização de modelós lineares e quadrados mínimos ponderados para anālise de tabelas de contingência. Essa proposição estā baseada no trabalho de: BHAPKAR (1966) que mostra a equivalência da estatística de WALD e a estatística de NEYMAN sendo que os fundamentos teóricos estão em WALD (1943).

A solução usual do $x^{2}$ de Pearson não possibili ta a partição de hipóteses, o que, é possível com o método GSK que permite o estudo do efeito isolado de cada fator.

A grande quantidade de produtos e inversões de matrizes que está relacionada com a metodologia do método GSK exige um programa de computador conveniente.

LANDIS et alii (1976), desenvolveram um progra ma para anālise de dados categorizados multivariados que é de nominado GENCAT.

Outros programas, também têm como finalidade a anālise de dados categorizados através do método estudado. 0 único inconveniente é que são programas para computador de grande porte.

Constitui objetivos deste trabalho desenvolver os conceitos teóricos de modo a tornar viāvel a programação para micro-computadores e principalmente, ajustar uma superfí cie de resposta a um experimento odontológico. 
0 experimento é constituido de cinco fatores (Idade, Tratamento, Sexo, Maxilar e Número de Raízes) com dois niveis cada um e existe interesse em se estudar a influência dos fatores, bem como suas possíveis interações, sobre a variảvel de anālise (reatividade).

Pelo exposto, adotamos um modelo fatorial $2^{5}$ e desse modo, os dados da amostra ficam distribuidos dentre trinta e duas sub-populações.

Alguns autores apresentaram preocupações sỏbre a validade do método para pequenas amostras. Com essas preocu pações adotamos uma amostra de $\mathrm{N}=2529$ elementos. 
2. REVISÃO DA LITERATURA

WALD (1943), estudou o problema de testar hipó teses constituídas por funções, que possuem derivadas parciais: contínuas de segunda ordem. Para testar tais hipóteses, propôs a estatística $W$ baseada em estimadores de máxima veros similhança. Demonstrou ainda que a estatística $W$ tem distribuição de $x^{2}$. central, sob $\mathrm{H}_{0}$.

NEYMAN (1949), apresentou um estudo sobre as propriedades e métodos de obtenção de estimador $x_{1}^{2}$ modifica do. Verificou que os estimadores $x_{1}^{2}$ modificados, são mais fáceis de serem obtidos do que os estimadores de máxima veros similhança quando as funções envolvidas são lineares, para os casos em que as funções não são lineares,o autor fornece um método de linearização, tornando possível a obtenção de esti- 
madores através de um sistema de equações lineares.

Demonstrou ainda NEYMAN que as estatísticas $x^{2}$ e $x_{1}^{2}$, usando estimadores de mäxima verossimilhança ou esti madores $\chi_{1}^{2}$ modificado, são consistentes e equivalentes ao tes te da razão de verossimilhança e, além disso, possuem distribuição assintōtica de $x^{2}$.

BHAPKAR (1966), adaptou a teoria de WALD a dados categóricos, sob o "modelo geral de classificação cruzada" usando uma variāvel dicotômica, tal que, a variāvel aøsumia valor 1 (um) se uma observação tivesse determinado atribu to e 0 (zero) nos demais casos. Mostrou ainda a equivalência algébrica entre a estatística de WALD e a estatística de NEYMAN, para hipöteses lineares sobre dados categorizados. Pa ra hipóteses não lineares estas se equivalem, desde que sejam linearizadas através de técnica proposta por NEYMAN.

Em estudo anterior, BHAPKAR (1961) descreveu a estatística de NEYMAN para testar hipóteses lineares, obtida pela minimização da estatística $x_{1}^{2}$ sujeita a restrições.

Pelo exposto, os testes de hipóteses lineares e não lineares são possíveis utilizando a estatística de WALD, que através da equivalência demonstrada pór BHAPKAR pertence à classe dos testes $x_{1}^{2}$ modificado e com distribuição assintótica de $x^{2}$, sob Ho.

BERKSON (1968), mostrou que para muitos proble mas o logit mínimo resulta, numericamente, as mesmas estimatí vas obtidas através de testes estatísticos como máxima verossimilhança e $x^{2}$ de Pearson. GRIZZLE et alli (1969) mostraram 
que o logit mínimo em grandes amostras possue distribuição de $x^{2}$ sendo Ho verdadeira, ou seja, é mais um membro desta família de testes.

BHAPKAR e KOCH (1968a), analisaram a existência de quatro tipos principais de tabelas, levando-se em conta "fatores" tais como blocos, tratamento e "respostas" como categorias de respostas. O primeiro tipo são as tabelas multi respostas, nenhum fator; o segundo são as tabelas multi-respostas, um fator; o terceiro são as tabelas de multi-respostas, multi-fatores e o quarto são as tabelas uma resposta, multi-fatores.

Para cada tipo de tabela o conceito de "não interação" está relacionado com as associações entre as "respostas" ou as combinações entre os "fatores". Os embasamentos teóricos dos testes estatísticos estão em WALD (1943).

GOODMAN (1968), diferenciou dois tipos de tabe las de contingência: (A) tabelas de contingência truncada, nas quais as frequências de algumas caselas são omitidas da anālise; e (B) tabelas de contingência não truncada, ou seja, as mais usuais em que a frequência de nenhuma casela é omitida quando da análise. Desta maneira, vārios métodos de análise de um tipo de tabela, quando modificado apropriadamente, torna-se adequado à analise do outro tipo.

$\mathrm{Na}$ prātica as tabelas não truncadas comumente são analisadas através do $x^{2}$ de Pearson. GOODMAN sugeriu novos procedimentos para análise. Esses métodos podem ser usados 
para testar hipóteses de nulidade de que as populações multinominais são quase homogêneas e podem ainda estimar proporções hipotéticas para as caselas, se nenhuma delas for nula. GRIZZLE, et alii (1969), formularam um mëtodo, que a partir daqui chamaremos método GSK, que ajusta funções a un modelo linear para resolver problemas que aparecem em tabelas de classificação cruzada, sob "o modelo geral de clas sificação cruzada", isto é, quando $\underline{s}$ amostras são retiradas de $\underline{s}$ populações e os elementos de cada amostra classifićados em $\underline{r}$ categorias de respostas. Baseia o desenvolvimento do método nas mesmas considerações feitas para a adaptação da estą tística de WALD a dados categorizados.

Descrevem ainda um procedimento para testar hipóteses sobre os parâmetros do modelo linear e para testar o ajuste do modelo aos dados.

Neste estudo são citados alguns exemplos de co mo o método pode ser usado para analisar vários tipos de da dos categorizados.

JONHSON e KOCH (1971), mostraram uma análise análoga ao "stepwise" usados em regressões com dados paramétricos e que baseia-se no uso de quadrados minimos ponderados para adptar modelos adequados a dados não paramétricos contidos em tabelas de contingência multi-dimensional.

GOODMAN (1971b), mostra usando um método análo go em alguns pontos ao "stepwise" na análise de regressão, co mo selecionar modelos adequados para descrever dados contidos em tabelas de $\underline{m}$ variāveis (ou fatores) e as possíveis intera- 
ções multiplicativas entre elas. Aponta ainda dificuldades pa ra aplicação do mētodo GSK quando para cada hipótese a ser testada hā necessidade de mudança nas matrizes envolvidas.

GRIZZLE e WILLIAMS (1972) baseados na equivalência entre a anālise fatorial e testes de independência mar ginal formularam prova capaz de testar independências de tabe las de contingência multi-dimensionais. OGLIARI (1984) faz ressalvas sobre algumas fórmulas e hipóteses do artigo.

WILLIAMS e GRIZZLE (1972), apresentaram doìs mé todos para análise de dados categorizados e os resultados de cada anālise são aparesentados na forma de análise de variân cia e adaptaram o método GSK ao modelo escalar de Thurstone.

LANDIS et alii (1976), desenvolveram um programa de computador que denominaram de GENCAT e que condensa toda metodologia que envolve análise de dados categorizados multi-variados. Esse algorítimo obtém estatísticas de $x_{1}^{2}$, modificado a partir de partições das somas de quadrados, como na anālise de variância paramética.

WADA (1977) conclui que a metodologia proposta por GRIZZLE et alii (1969) apresenta vantagens sobre as soluções usuais e que, apesar de muitas dificuldades, apontadas por diversos pesquisadores na aplicação do método GSK, de um modo geral esse procedimento é de fácil aplicação.

OGLIARI (1984), fez estudo sobre o problema causado pela aproximação assintótica dos testes utilizados em tabelas de contingência.

Desenvolveu um programa de anālise em linguagem 
"BASIC" de modelos log lineares aos dados de tabela de contin gência do tipo CxV́F e a determinação de estruturas de independência para os dados dessas tabelas.

Conclui que para amostras entre 1.000 e 1.500 dados os resultados são bastantes eficientes no sentido de in dicar um modelo que represente a estrutura de independência dos dados das tabelas. 
3. METODOLOGIA

3.1. Metodologia GSK

Considere $s$ amostras simples e independentes de tamanho $n_{1}, n_{2} \ldots n_{s}$ extraídas de $\underline{s}$ distribuições multino miais (consideradas aqui como sub-ipopulações).

Para efeito teórico os $\mathrm{n}_{i}$ serão fixos à priori. Cada uma das distribuições acima apresenta $\underline{r}$ categorias de respostas distintas.

Os dados resultantes da amostragem podem ser resumidos numa tabela de contingência ( $\mathrm{s} \times r$ ), onde $\mathrm{n}_{i j}$ denota a frequência de respostas da j-ésima categoria na amostra da i-ésima sub-população. 
TABELA 1: Distribuição de frequêencia segundo categoria de res postas e sub-populações.

SUB POPULAÇOES

CATEGORIAS DE RESPOSTAS

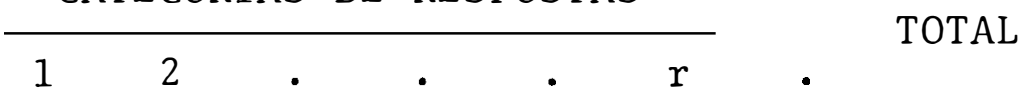

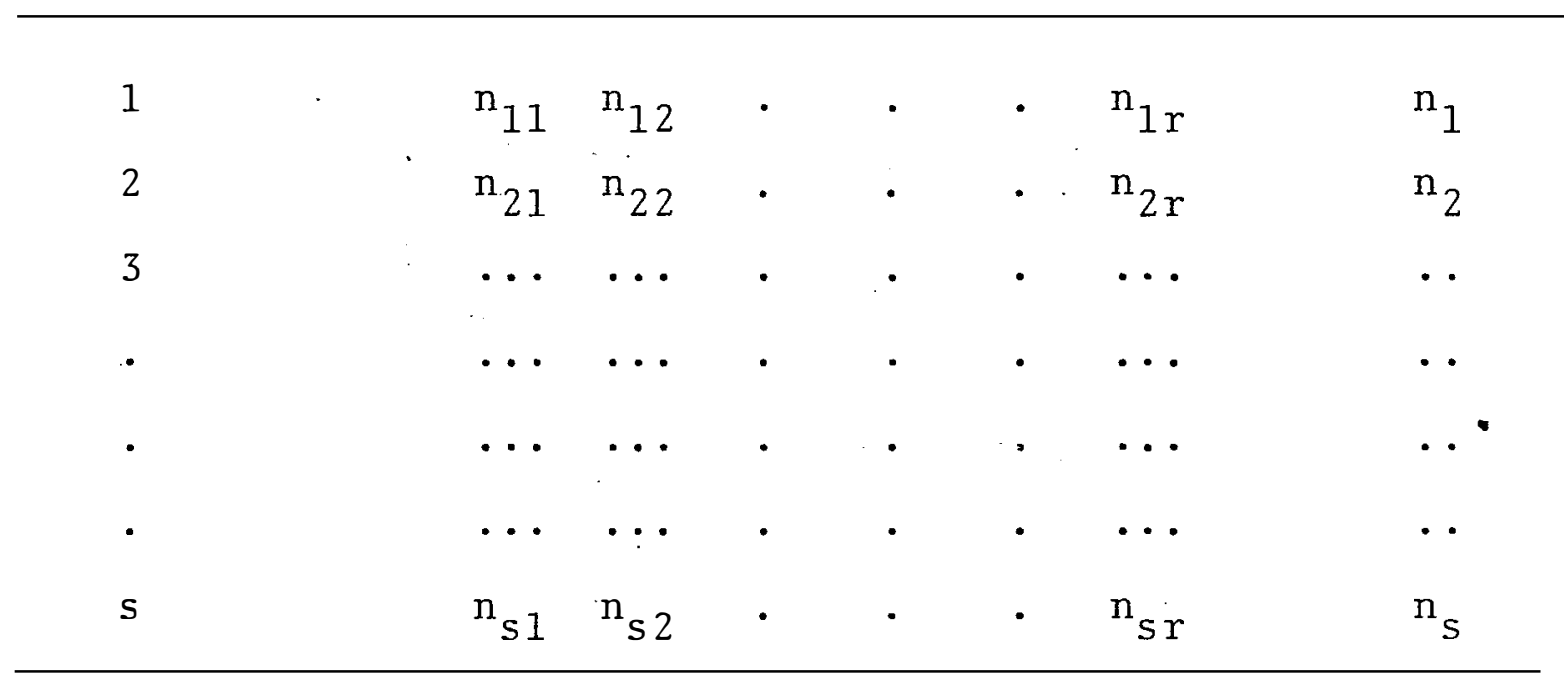

o Vetor aleatório $n_{i} \operatorname{com} i=1,2, \ldots s$, segue por hipótese uma distribuição multinomial com parâmetros ${\underset{\sim}{i}}_{i}$ e $\underline{\sim}_{i}$, onde $\underline{\sim}_{\sim} i$ é um vetor $\operatorname{rxl}$ e $\pi_{i j}$ representa a probabilidade de que um elemento selecionado ao acaso, da i-ésima sub população, seja classificado na j-ésima categoria de resposta.

Vetores ${\underset{\sim}{i}}_{i}$ e $\stackrel{\pi}{i}$

$$
\stackrel{\sim}{n}_{i}^{\prime}=\left(\begin{array}{c}
n_{i 1} \\
n_{i 2} \\
\cdot \\
n_{i r}
\end{array}\right) \quad \underline{\sim}_{i}^{\prime}=\left(\begin{array}{c}
\pi_{i 1} \\
\pi_{i 2} \\
\cdot \\
\pi_{i r}
\end{array}\right)
$$


Vetor aleatório é um Vetor cujos componentes são variáveis aleatórias. Seja $X$ um ponto de coordenadas $\mathrm{X}\left(\mathrm{x}_{1}, \mathrm{x}_{2}, \ldots \mathrm{x}_{\mathrm{m}}\right)$. A função de probabilidade conjunta da mul tinomial em $x$ é:

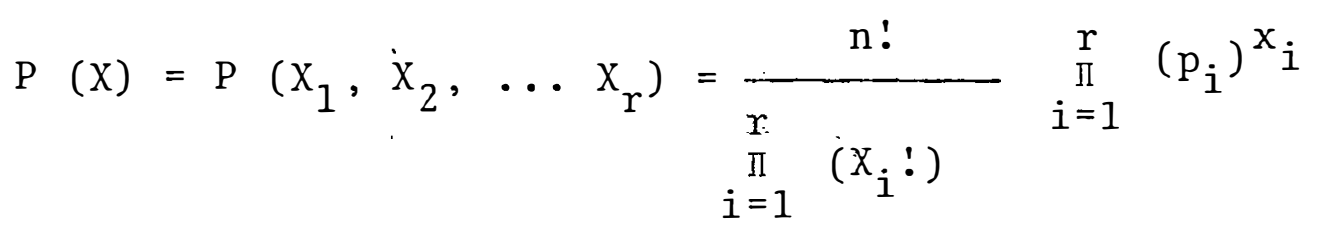

$$
\begin{aligned}
\operatorname{com} . & \sum \mathrm{X}_{\mathrm{i}}=\mathrm{n} \\
\mathrm{i} & =1,2, \ldots, \mathrm{r} \\
0 & \leq \mathrm{p}_{\mathrm{i}} \leq 1
\end{aligned}
$$

Esta fórmula è uma generalização da binomial, onde :

$P(X)=\frac{n !}{r !(n-r) !} \quad p^{r}(1-p)^{n-r}$ onde por analogia podemos fazer

$x\left(x_{1}, x_{2}\right)$ e $P(X)=\frac{n !}{x_{1} ! x_{2} !} p_{1}^{x_{1}} p_{2}^{x_{2}}$

$$
\therefore P(x)=P\left(x_{1}, x_{2}\right)=\frac{n !}{\prod_{i=1}^{2}\left(x_{i} !\right)} \underset{i=1}{2} \prod_{i}^{x_{i}}
$$

Considerando-se a tabela anterior, podemos escrever que a probabilidade do i-ésimo Vetor, é: 


$$
P\left(n_{i}\right)=\frac{n_{i} !}{\prod_{j=1}^{r}\left(n_{i j}\right) !} \prod_{j=1}^{r} \quad\left(\pi_{i j}\right)^{n_{i j}}
$$

Este Vetor de probabilidade corresponde a i-és $\underline{i}$ ma sub-população multinomial.

Como as sub-populações são independentes entre si, os $\underline{s}$ vetores serão linearmente independentes e o seu produto que no caso é o modelo matemático do problema será:

$\Phi=\prod_{i=1}^{s}\left(n_{i}:\right) \underset{j=1}{r}\left(\frac{n_{i j}^{n_{i j}}}{n_{i j} !}\right)$

Com as restrições

$$
\sum_{j=1}^{r} \pi_{i j}=1 \text { para todo } i=1,2, \ldots, s
$$

(cada linha é uma multinomial e portanto a soma de suas prob $\underline{a}$ bilidades deve ser 1)

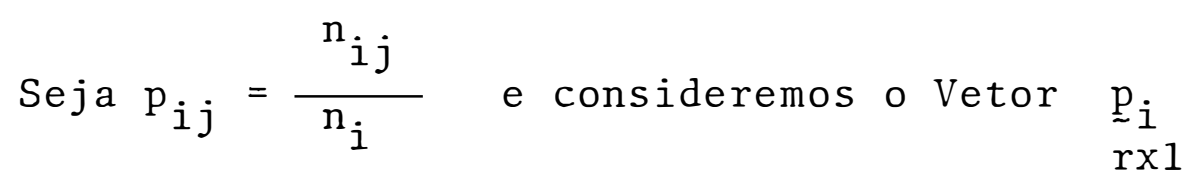

$\underset{r \times 1}{p_{i}}=\left(\frac{n_{i 1}}{n_{i}}, \frac{n_{i 2}}{n_{i}}, \ldots, \frac{n_{i r}}{n_{i}}\right)$ com $i=1,2, \ldots, s$

Cada Vetor $\mathrm{p}_{i}$ é um estimador da verdadeira pro babilidade. Portanto $\underline{p}_{i}$ é um Vetor rxl das proporções observa 
das associadas com as amostras da i-ésima sub-população. Seja agora o Vetor $\underset{\sim}{\mathrm{p}}$ de dimensões rsxl que contém todas as proporções de todas as $\underline{s}$ distribuições multinomiais

$\underset{\tilde{r} s \times 1}{p^{\prime}}=\left(\underline{\sim}^{\prime} 1, \underset{p^{\prime}}{\prime} 2, \ldots \underline{\sim}^{\prime} s\right)$

O Vetor $\underset{\sim}{p}$ é um estimador de máxima verossimilhança, sem estar sujeito a qualquer hipōtese Ho e sem restrições, de $\pi$ onde

$\pi_{\tilde{r} S \times 1}^{i}=\left(\pi i, \pi i, \ldots, \pi_{s}^{i}\right)$

Destacando que em cada casela temos

$p_{i j}=\hat{\pi}_{i j}=\frac{n_{i j}}{n_{i}}$

Como estamos considerando s distribuições multinomiais hả correlação entre os elementos da mesma distribui ção (no caşo entre elementos da mesma linha), mas, .não hã en tre as distribuições jā que são duas a duas independentes.

MATRIZ DE VARIÂNCIAS E COVARIÂNCIAS

Consideremos a i-ésima multinomial, podemos ob ter a seguinte matriz considerando-se as variâncias na diagonal e as covariâncias fora dela. Observamos, ainda, que o ele mento estā na j-ésima casela ou fora dela e assim podemos raciocinar como em uma binomial 


$$
\begin{aligned}
& V\left(\frac{n_{i j}}{n_{i}}\right)=\frac{1}{n_{i}^{2}} \cdot V\left(n_{i j}\right) \quad V\left(n_{i j}\right)=n_{i} \pi_{i j}\left(1-\pi_{i j}\right) \\
& . V\left(\frac{n_{i j}}{n_{i}}\right)=\frac{1}{n_{i}} n_{i} \pi_{i j}\left(1-\pi_{i j}\right)= \\
& V\left(\frac{n_{i j}}{n_{i}}\right)=\frac{\pi_{i j}\left(1-\pi_{i j}\right)}{n_{i}}
\end{aligned}
$$

\section{Ainda na i-ésima multinomial temos as covariān}

cias

$\operatorname{Cov}\left(\frac{n_{i j}}{n_{i}}, \frac{n_{i j}}{n_{i}}\right)$

Consideremos

$\operatorname{Var}\left(\frac{n_{i j}+n_{i j}{ }^{\prime}}{n_{i}}\right)=\operatorname{Var}\left(\frac{n_{i j}}{n_{i}}\right)+\operatorname{Var}\left(\frac{n_{i j}{ }^{\prime}}{n_{i}}\right)+2 \operatorname{Cov}\left(\frac{n_{i j}}{n_{i}}, \frac{n_{i j}}{n_{i}}\right)$

$\operatorname{Cov}\left(\frac{n_{i j}}{n_{i}}, \frac{n_{i j}{ }^{\prime}}{n_{i}}\right)=\frac{1}{2}\left[V\left(\frac{n_{i j}+n_{i j}^{\prime}}{n_{i}}\right)-V\left(\frac{n_{i j}}{n_{i}}\right)-V\left(\frac{n_{i j}}{n_{i}}\right)\right]$

$\operatorname{Var}\left(\frac{n_{i j}+n_{i j}^{\prime}}{n_{i}}\right)=\frac{1}{n_{i}^{2}} V\left(n_{i j}+n_{i j}^{\prime}\right)$

onde $V\left(n_{i j}+n_{i j}{ }^{\prime}\right)=n_{i}\left(\pi_{i j}+{ }^{\prime} \pi_{i j}{ }^{\prime}\right)\left(1-\pi_{i j}-\pi_{i j}{ }^{\prime}\right)$

$\cdot \operatorname{Var}\left(\frac{n_{i j}+n_{i j}{ }^{\prime}}{n_{i}}\right)=\frac{\left(\pi_{i j}+\pi_{i j}{ }^{\prime}\right)\left(1-\pi_{i j}-\pi_{i j}{ }^{\prime}\right)}{n_{i}}$ 
então

$\operatorname{Cov}\left(\frac{n_{i j}}{n_{i}}, \frac{n_{i j}}{n_{i}}\right)=\frac{1}{2}\left[\frac{1}{n_{i}}\left(\pi_{i j}+\pi_{i j} i\right)\left(1-\pi_{i j}-\pi_{i j}\right)-\right.$

$$
\left[-\frac{1}{n_{i}} \pi_{i j}\left(1-\pi_{i j}\right)-\frac{1}{n_{i}} \pi_{i j},\left(1-\pi_{i j}{ }^{\prime}\right)\right]
$$

Desenvolvendo e simplificando a expressão obte

mos:

$\operatorname{Cov}\left(\frac{n_{i j}}{n_{i}}, \frac{n_{i j} n_{i}}{n_{i}}=-\frac{\pi_{i j} \pi_{i j}}{n_{i}}\right.$

Portanto a matriz de Variância-covariância da i-ēsima multinomial fica:

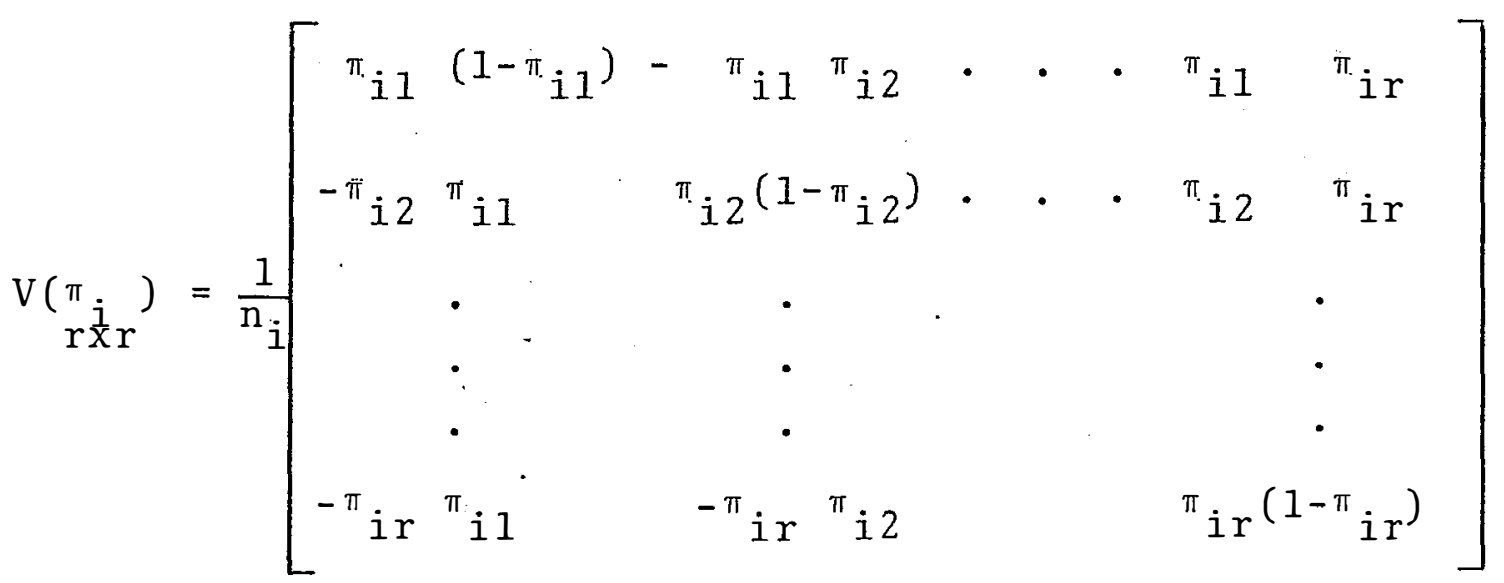

A matriz $\underset{\operatorname{rxr}}{V\left(p_{i}\right)}$ é um estimador de $\left.\underset{\operatorname{rxr}}{V(\pi}{ }^{\mathrm{i}}\right)$

Como estamos estimando probabilidades, a amostra deve ter um tamanho suficiente.

$$
\text { Pode-se dizer que } p_{i j}=\frac{n_{i j}}{n_{i}} \text { é um estimador }
$$


consistente para ${ }^{i}{ }_{i j}$ quando $n_{i} \rightarrow \infty$

$\mathrm{Na}$ prātica, todavia, com valores não muito grande de $n_{i} j a ̄$ podemos desenvolver as aplicações, que a teoria funciona.

A matriz com todas as informações sobre variân cias e covariâncias é dada por:

$\underset{\tilde{r} s \tilde{x} r s}{V(\pi)}=$ Matriz bloco diagonal com elementos $\underset{\operatorname{rxr}}{V\left(\pi_{i}\right)}$ na diagonal principal e zeros fora dela, devido à independência entre as linhas (independência duas a duas das multinomiais).

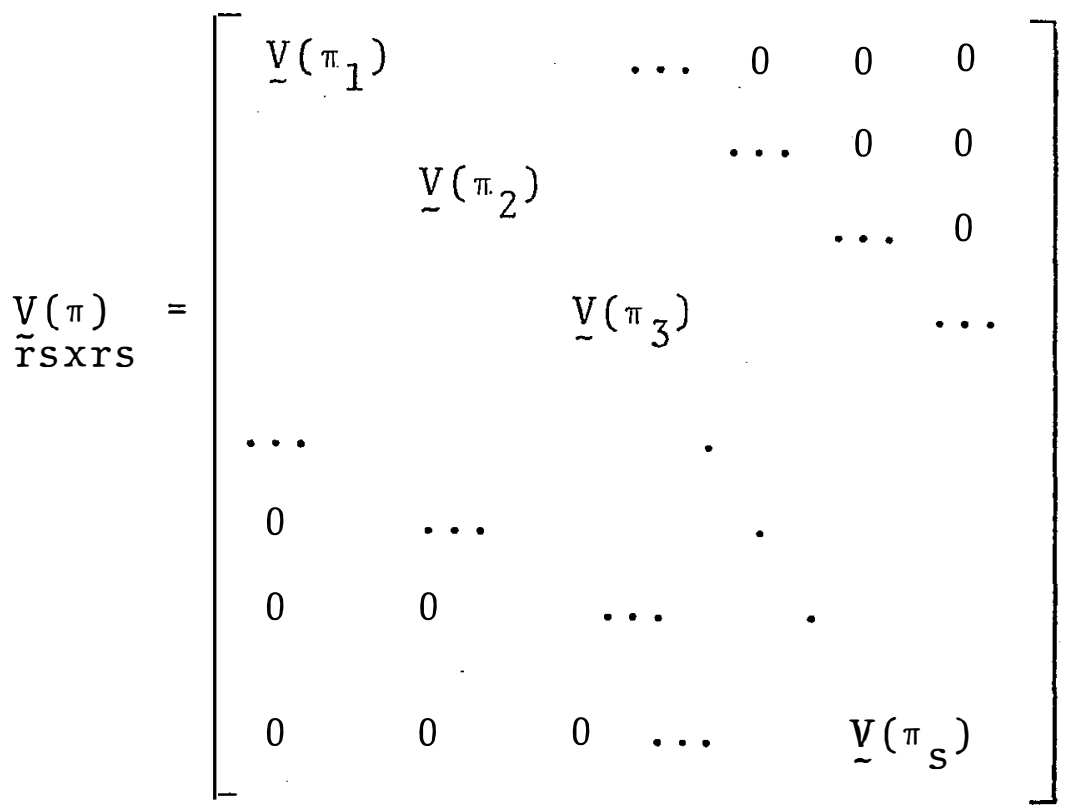

A matriz $\underset{\bar{r} \text { (pxs }}{V}$ é um estimador consistente da matriz de variâncias e covariâncias'( $\pi)$ onde $\pi$ é um Vetor rsxl rsxrs

contendo todas as proporções teóricas e o vetor $\underset{\sim}{p}$ que o estima contēm todas as proporções $\frac{n_{i j}}{n_{i}}$ observadas.

Sejam as funções $F_{1}(\underline{p}), F_{2}(\underline{p}) \ldots, F_{u}(\underline{p})$ com 
usrs-s pois hâ rs elementos com uma restrição em cada multino mial isto é:

Cada linha i tem $\mathrm{r}-1$ graus de liberdade.

No total teremos $s(r-1)=s r-s$ graus de $1 \underline{i}$

berdade.

$\{\mathrm{F}(\mathrm{p})\} \overrightarrow{\mathrm{e}}$ um conjunto de $\underline{u}$ funções que descrevem em algum aspecto a relação entre as distribuições das res postas e a natureza das sub-populações consideradas.

Cada uma dessas funções tem, por hipótese, "derivadas parciais contínuas até 2 a ordem, com respeito aos ele mentos de $\underline{p}$, dentro de uma região aberta contendo $\underset{\sim}{\pi}=E(\underline{p})$

Seja $\underset{\mathrm{uxl}}{\mathrm{F}^{\prime}}=\underset{\sim}{\mathrm{F}}(\underline{p})=\left[F_{1}(p), F_{2}(p) \cdot . \cdot F_{u}(p)\right]$

Desta forma, um estimador consistente da matriz de Variâncias e Covariâncias do Vetor $\mathrm{F}$ è dado por $\mathrm{S}$ = $\mathrm{HV}(\mathrm{p}) \mathrm{H}^{\prime}$ onde

$\underset{\text { uxrs }}{H}=\left[\begin{array}{lll}\frac{\partial}{\underline{F}_{m}} & (\underset{\sim}{(j)}) \\ \hline \pi_{i j}\end{array}\right]_{\pi_{i j}}=p_{i j}$

com $i=1, \ldots, s ; j=1, \ldots, r ; m=1, \ldots, u$

Se $\underset{\sim}{F}$ for linear: $\underset{\tilde{u} \times l}{F}=\underset{\tilde{u} \times r s}{A} \underset{\widetilde{r} S \times l}{\mathrm{P}}$

$\frac{\partial \mathrm{F}}{\partial \mathrm{p}}=\underset{\sim}{\mathrm{A}}=\underset{\sim}{\mathrm{H}}$

Se $\underset{\sim}{F}$ for não linear 
$F(\underline{\pi})=\underline{F}(\underline{p})+\left[\frac{\partial F(\pi)}{\partial \pi}\right]=p(\underline{\sim}-\underline{p})+E_{n}|| \underset{\sim}{\pi}-p||$

onde $\mathrm{H}=\left[\frac{\mathrm{F}(\pi)}{\partial \pi}\right]_{\pi=\mathrm{p}}=\mathrm{K} \mathrm{D}^{-1} \mathrm{~A}$

e $D_{a}$ é uma matriz diagonal com elementos do Vetor a $=A p$

Para funções aproximadamente lineares $y=\psi(x)$ usa-se o desenvolvimento de Taylor. Assim:

$y=\psi(x)=\psi(\xi)+(x-\xi) \psi^{\prime}(\xi)$

$V(y)=\left[\psi^{\prime}(\xi)\right]^{2} \quad \mathrm{~V}(\mathrm{x})$, onde $\mathrm{HH}^{\prime}$ corresponde $\mathrm{a}\left[\psi^{\prime}(\xi)\right]^{2}$

$\pi_{i j}=x ; p_{i j}=\xi$ e $\frac{\partial F_{m}(\pi)}{\partial \pi_{i j}}=\psi^{\prime}(\xi)$

Nas aplicações as funções $\operatorname{Fm}(\underset{-}{\pi})$ para $m=1,2$, ... u, devem ser escolhidas de sorte que $S$ (matriz de variâncias - covariâncias), seja assintoticamente não-singular) .

$\underset{\sim}{\mathrm{F}}(\underset{\mathrm{p}}{)}$ é um estimador consistente de $\underset{\sim}{\mathrm{F}}(\underset{\sim}{\pi})$. Conse quentemente a variação entre os elementos de $\underline{F}(\pi)$ pode ser in vestigada, pelo ajustamento de modelos de regressão linear, por meio do método dos mínimos quadrados ponderados. Isto pode ser caracterizado por meio da relação:

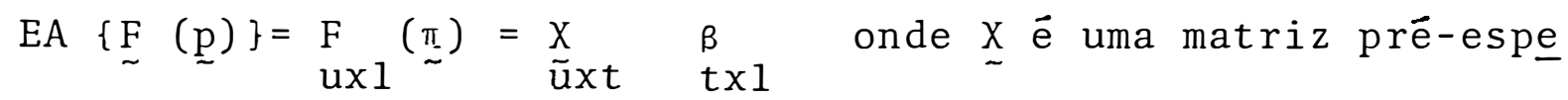
cificada (uxt) de planejamento de elementos conhecidos e o posto de $X \bar{e} t \leq u, \underline{B}$ é um vetor $(t x 1)$ de parâmetros desconhe cidos e EA denota o valor assintótico esperado de $\underset{\sim}{F}(\underline{p})$. 
Por hipötese as funções $\mathrm{Fm}(\pi)$ para $\mathrm{m}=1,2$, ..., u são independentes e também independentes das restri-

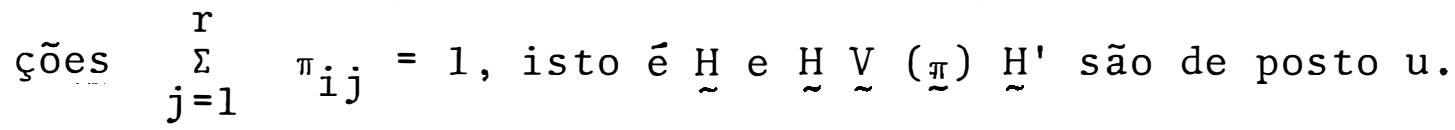

Contudo para alguns tipos de dados se algum $n_{i j}=o, \quad s$ será de posto menor que u.

Neste caso, usaremos procedimento proposto por BERKSON (1955), que substitui os valores zeros por $1 / \mathrm{r}$. Is to implica que $\hat{\pi}_{i j}=p_{i j}=\frac{1}{\text { rni }}$ para 0 valor substituído.

\section{EST IMAÇAO E TESTES}

Até este ponto foram definidas ú funções paramétricas de $\pi, F m(\pi), m=1,2, \ldots u$, possivelmente não line a res, suas estimativas $F m(p)$ e sua matriz de variância - covariância $S=\underset{\sim}{H} \underset{\sim}{H}(p) \underset{\sim}{H}$ '

O modelo é linear: $\underset{\sim}{\operatorname{EA}}\{\mathrm{F}(\mathrm{p})\}=F(\pi)=\underset{\sim}{\mathrm{X}} \underset{\sim}{\beta}$

Se o modelo hipotético ajusta-se "bem" aos dados, o melhor estimador assintótico de $\beta$ é dado por $\underline{b}$ onde: $\underset{\tilde{\mathrm{E}} \times 1}{\mathrm{~b}}=\left(\underset{\sim}{X} S^{-1} \underset{\sim}{X}\right)^{-1} \underset{\sim}{X} S^{\prime} \quad S^{-1} \underset{\sim}{F}$

b é o melhor estimador de $\beta$ (BAN - best assimptot normal)

b é obtido minimizando a forma quadrātica (mínimos quadrados generalizados) 
$\min (\underset{\sim}{F}-\underset{\sim}{X})^{\prime} S^{-1}(\underset{\sim}{F}-\underset{\sim}{X} \underset{\sim}{\beta})=(\underset{\sim}{F}-\underset{\sim}{X} \underset{\sim}{b})^{\prime} S^{-1}(\underset{\sim}{F}-\underset{\sim}{X b})$

O teste estatístico usado para verificar

o

ajustamento do modelo é dado por:

$S Q\{F(\pi)=X \beta\}=\underset{1 \tilde{x} u}{F^{\prime}} \underset{u^{-1}}{S^{-1}} \underset{u \tilde{x} 1}{F}-\underset{1 \tilde{x} t}{b^{\prime}}\left(\underset{t \tilde{x} u}{X^{\prime}} \underset{\text { uxu }}{S^{-1}} \underset{u \tilde{x} t}{X}\right)_{t \tilde{x} 1}^{b}$

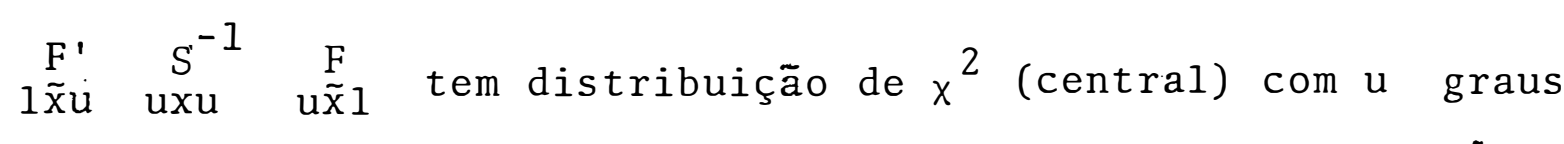
de liberdade.

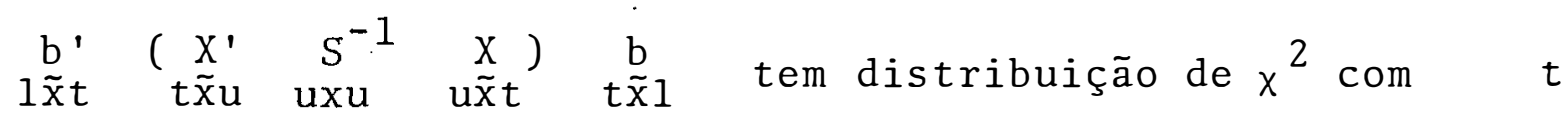
graus de liberdade.

Os $x^{2}$ ocorrem assintoticamente. O resultado é portanto uma distribuição de $\chi^{2} \operatorname{com}(u-t)$ graus de liberdade.

Se o modelo $\underset{\sim}{F}\left(\pi_{\sim}\right)=\underset{\sim}{X} \underset{\sim}{\operatorname{B}}$ aceito, podemos tes tar hipóteses da forma Ho: $\underset{\sim}{C} \underset{\sim}{\beta}=\underset{\sim}{0}$ onde $\underset{\sim}{\mathrm{C}}$ uma matriz c $\times \mathrm{t}$ com $c \leq t$ e $\underset{0}{e}$ é vetor nulo cxl.

o teste estatístico é dado por:

$Q C=S Q\{C \beta=0\}=(C b)^{\prime}\left\{C\left(X^{\prime} \quad S^{-1} X\right)^{-1} C^{\prime}\right\}^{-1}$

que é assintoticamente uma distribuição de $x^{2}$ com $\underline{c}$ graus de liberdade se Ho é verdadeira.

0 posto de $\mathrm{C}$ é $\underline{c}$. A estimativa de $\mathrm{C}_{\beta}$ é $\mathrm{Cb}$.

Quando Ho é verdadeira $\mathrm{C} B=0 \Longrightarrow \mathrm{Cb}=0$, onde, $V(b)=\left(X^{\prime} \quad S^{-1} X\right)^{-1}$

e

$\mathrm{V}(\mathrm{Cb})=\mathrm{C} \operatorname{Var}(\mathrm{b}) \mathrm{C}^{\prime}=\mathrm{C}\left(\mathrm{X}^{\prime} \mathrm{S}^{-1} \mathrm{X}\right)^{-1} \mathrm{C}^{\prime}$. 


\subsection{Tabelas de Classificacão Cruzada mais Usuais.}

Os dados categorizados são frequentemente apre sentados em dois tipos de tabelas de classificação cruzada; um com dados provenientes de uma população e outro com dados provenientes de vārias populações.

No primeiro tipo, os dados são apresentados em tabelas de contingência onde $\underline{N}$ elementos de uma amostra são classificados em dois atributos. 0 número de categorias de respostas podem ser igual ou diferente para cada atributo. No caso de igualdade teremos uma tabela quadrada. 
TABELA 2: DISTRIBUIÇÃO DE FREQUENCIA POPULACIONAL SEGUNDO AS CATEGORIAS DE RESPOSTAS DE DOIS ATRIBUTOS

\begin{tabular}{|c|c|c|c|c|c|c|c|}
\hline \multirow{2}{*}{ 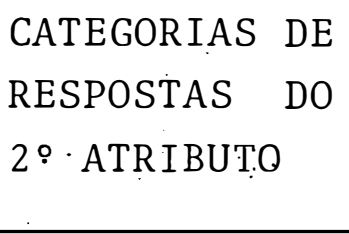 } & \multicolumn{6}{|c|}{ CATEGORIAS DE RESPOSTAS DO 10 ATRIBUTO } & \multirow{2}{*}{ TOTAL } \\
\hline & 1 & 2 & . & . & . & $\mathrm{r}$ & \\
\hline 1 & $\mathrm{n}_{11}$ & $\mathrm{n}_{12}$ & • & • & $\cdot$ & $n_{i r}$ & $\mathrm{n}_{1}$. \\
\hline 112 & $\mathrm{n}_{21}$ & $\mathrm{n}_{22}$ & - & • & $\cdot$ & $\mathrm{n}_{2 \mathrm{r}}$ & ${ }^{\cdot} \mathrm{n}_{2}$ \\
\hline$\cdots$ & $\cdots$ & $\cdots$ & - & $\cdot$ & $\cdot$ & $\cdots$ & $\cdots$ \\
\hline $\mathrm{r}$ & $\mathrm{n}_{\mathrm{r} 1}$ & $\mathrm{n}_{\mathrm{r} 2}$ & ${ }^{\circ}$ & $\cdot$ & • & ${ }^{n} \operatorname{rr}$ & $\mathrm{n}_{\mathrm{r}}$ \\
\hline TOTAL & $\mathrm{n} .1$ & $\mathrm{n}^{2} 2$ & $\cdot$ & • & • & ${ }^{n} \cdot r$ & $\mathrm{n}$ \\
\hline
\end{tabular}

Tabela quadrada $\underline{r}$ categorias de respostas uma população onde os $n_{i j}$ são as frequências da casela ( $i j$ ) e os $n_{i .}, n_{. j}$ são os totais marginais.

Para esses casos de amostras provenientes de uma única população, as hipóteses estatísticas apropriadas são formuladas por $\underset{\sim}{\mathrm{F}}\left(\underset{\sim}{\pi_{0}}\right)=0$, dispensando dessa maneira a cons trução da matriz $X$ referente ao planejamento.

0 teste estatístico apropriado para o ajuste resume-se a: $S Q \underset{\sim}{F}(\underset{\sim}{\pi})=0=\underline{F}^{\prime} S^{-1} \underset{\sim}{(F)} \underset{\sim}{F}$ que tem distribuição $x^{2}$ central assintótica com u graus de liberdade se Ho for verdadeira. 
Com o número de respostas $r=2$, para tabelas desse tipo, ficar fảcil verificar a distribuição do teste, como segue:

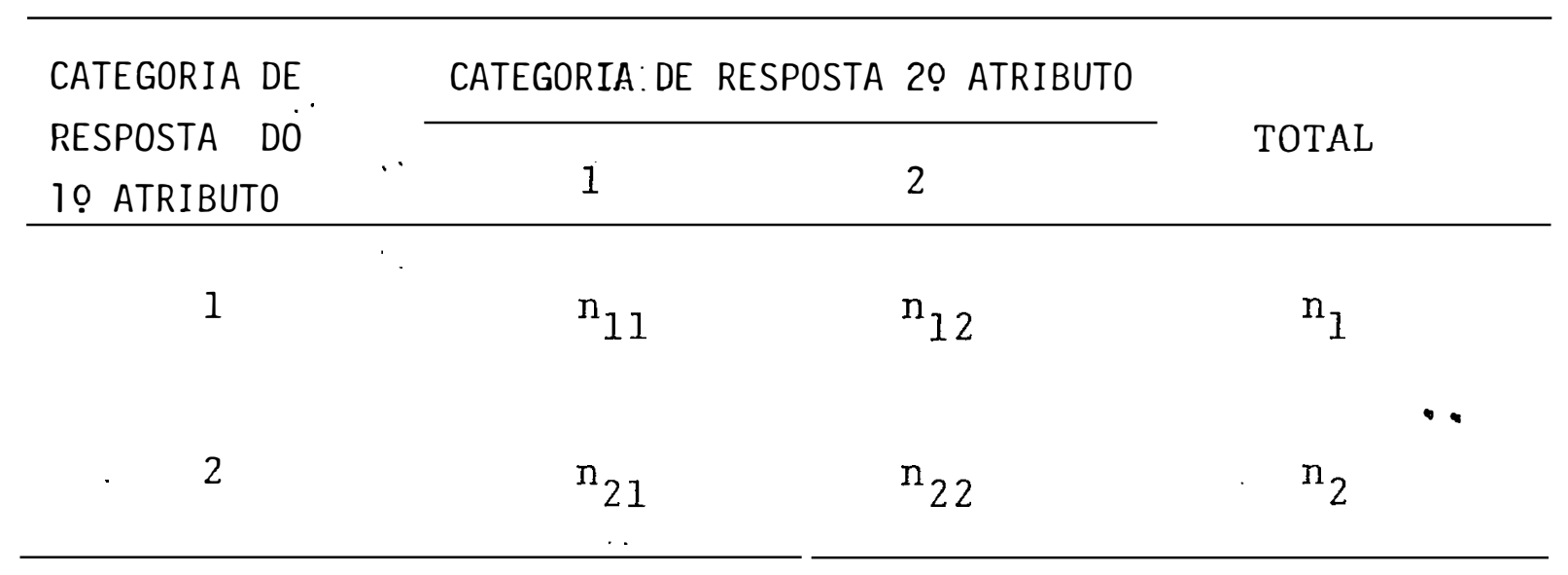

Suponhamos que, a hipótese a ser testada seja: Ho: $\pi_{11}=\pi_{21}$, o que implica que $\pi_{11}-\pi_{21}=0$.

\section{Então poderíamos escrever}

Ho: $1 \pi_{11}+0 \pi_{12}-1 \pi_{21}+0 \pi_{22}=0$

$\operatorname{Amatriz} \underset{4 \tilde{x} 1}{p}=\left[\begin{array}{l}p_{11} \\ p_{12} \\ p_{21} \\ p_{22}\end{array}\right]$

A matriz $\underset{1 \times 4}{A}=\left\{\begin{array}{llll}1 & 0 & -1 & 0\end{array}\right\}$

$\underline{F}(p)=A \times p$

$\underline{F}(p)=\left\{p_{11}-p_{21}\right\}$

$\underset{4 \times 4}{V(p)}=\left[\begin{array}{ccc}V\left(p_{1}\right) & 0 \\ 0 & V\left(p_{2}\right)\end{array}\right]$ 
$V\left(p_{1}\right)=\left[\begin{array}{l}\frac{p_{11}\left(1-p_{11}\right)}{n_{1}} \\ \frac{-p_{11} p_{12}}{n_{1}}\end{array}\right.$

$\left.\begin{array}{l}\frac{-p_{11} p_{12}}{n_{1}} \\ \frac{p_{12}\left(1-p_{12}\right)}{n_{1}}\end{array}\right]$

$V\left(p_{2}\right)=\left[\begin{array}{l}\frac{p_{21}\left(1-p_{21}\right)}{n_{2}} \\ \frac{-p_{21}\left(1-p_{21}\right)}{n_{2}}\end{array}\right.$

$\left.\begin{array}{l}\frac{-p_{21} \quad p_{22}}{n_{2}} \\ \frac{p_{22}\left(1-p_{22}\right)}{n_{2}}\end{array}\right]$

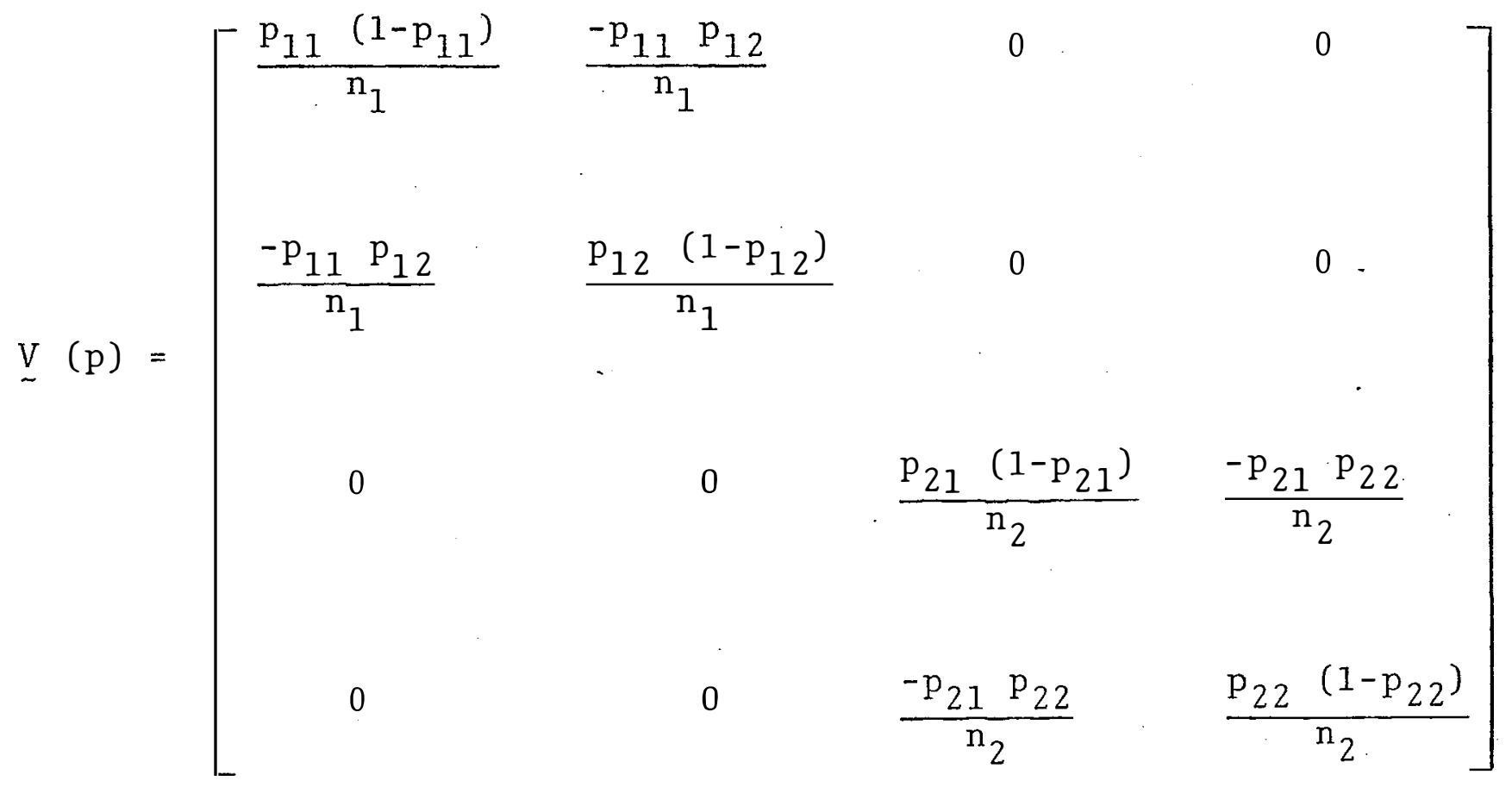




$$
S=A \underset{\sim}{V}(p) A^{\prime}=\left[\frac{p_{11}\left(1-p_{11}\right)}{n_{1}}-\frac{p_{21}\left(1-p_{21}\right)}{n_{2}}\right]
$$

$S Q\{F(\pi)=0\}=\underline{F}^{\prime} S^{-1} F$

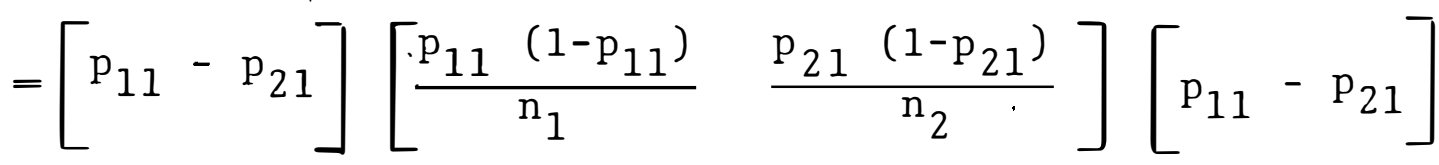

$S Q\left[F(\pi)^{\prime}=0\right]=\frac{p_{1} p_{2}\left(p_{11}-p_{21}\right)^{2}}{n_{2} p_{11}\left(1-p_{11}\right)+n_{1} p_{21}\left(1-p_{21}\right)}$

SQ F $(\pi)=0=\frac{\mathrm{n}_{1} \mathrm{n}_{2}\left[\frac{\mathrm{n}_{11}}{\mathrm{n}_{1}}-\frac{\mathrm{n}_{21}}{\mathrm{n}_{2}}\right]^{2}}{\mathrm{n}_{2} \frac{\mathrm{n}_{11}}{\mathrm{n}_{1}} \frac{\mathrm{n}_{12}}{\mathrm{n}_{1}}+\mathrm{n}_{1} \frac{\mathrm{n}_{21}}{\mathrm{n}_{2}} \frac{\mathrm{n}_{22}}{\mathrm{n}_{2}}}$

SQ $F(\pi)=0=\frac{n_{1} n_{2}\left(n_{2} n_{11}-n_{1} n_{21}\right)^{2}}{n_{2}^{3} n_{11} n_{12} n_{1}^{3} n_{21} n_{22}}$

(I) è a expressão de um $x^{2}$ com 1 grau de liberdade.

A anālise estatística de dados categorizados provindos de uma população, a exemplo do que consta da tabe1a 2, podem ser feita através das hipóteses:

a) Independência, formulada por Ho: $\pi_{i j}=\pi_{i \cdot}{ }^{\pi} \cdot j$

b) associação sobre a diagonal principal, formulada por Ho: $\pi_{i i}=\pi_{i \cdot} \cdot{ }^{\prime} \cdot j$

c) homogeneidade das marginais, formulada por, Ho: $\pi_{i} \cdot=\pi \cdot_{j}$ com $i=j=1,2, \ldots, r$ 
d) simetria, formulada por, Ho: $\pi_{i j}=\pi_{j i}$ com $i \neq j$

Passamos, agora a adaptar cada uma dessas hipó teses ao mëtodo "GSK". Para nos auxiliar, mostramos uma tabela de proporções teóricàs.

TABELA 3: PROPORÇÕES TEÓRICAS DE UMA TABELA DE CONTINGENCIA POPULACIONAL COM DOIS ATRIBUTOS

\begin{tabular}{|c|c|c|c|c|c|}
\hline \multirow{2}{*}{$\begin{array}{c}2^{\circ} \\
\text { ATRIBUTO }\end{array}$} & \multicolumn{4}{|c|}{$1^{\circ}$ ATRIBUTO } & \multirow{2}{*}{ TOTAL } \\
\hline & 1 & 2 & $\ldots$ & $\mathrm{r}$ & \\
\hline 1 & $\pi 11$ & ${ }^{\pi} 12$ & $\cdots$ & $\pi$ & $\pi$. \\
\hline 2 & $\pi 21$ & $\pi 22$ & $\cdots$ & ${ }^{\pi} 2 r$ & $\pi_{2}$. \\
\hline$\cdots$ & $\cdots$ & $\cdots$ & $\cdots$ & $\cdots$ & $\cdots$ \\
\hline$r$ & ${ }^{\pi} \mathrm{rl}$ & ${ }^{\pi} \mathrm{r} 2$ & $\cdots$ & ${ }^{\pi} \mathrm{rr}$ & ${ }^{\pi} \mathrm{r}$ \\
\hline TOTAL & $\pi .1$ & ". 2 & $\cdots$ & $\pi \cdot r$ & 1 \\
\hline
\end{tabular}

Essa hipótese pode ser testada em tabelas onde o número de categoria de respostas nos dois atributos, sejam iguais ou diferentes. No caso particular de uma tabela $3 \times 3$ te ríamos : 


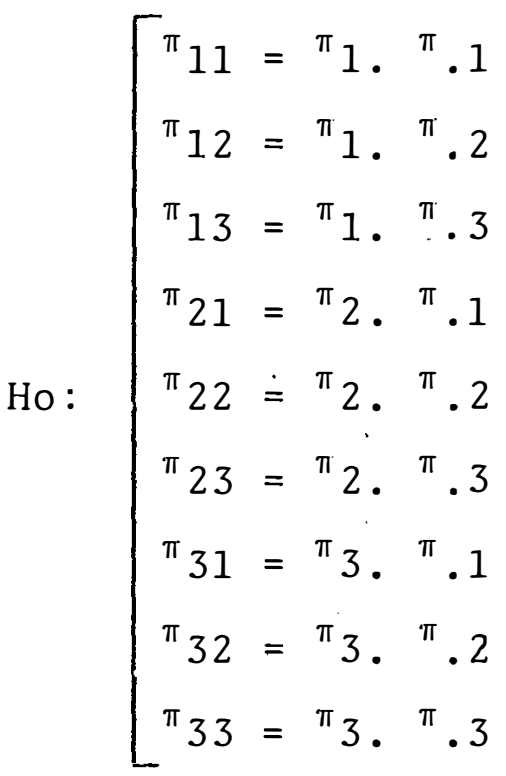

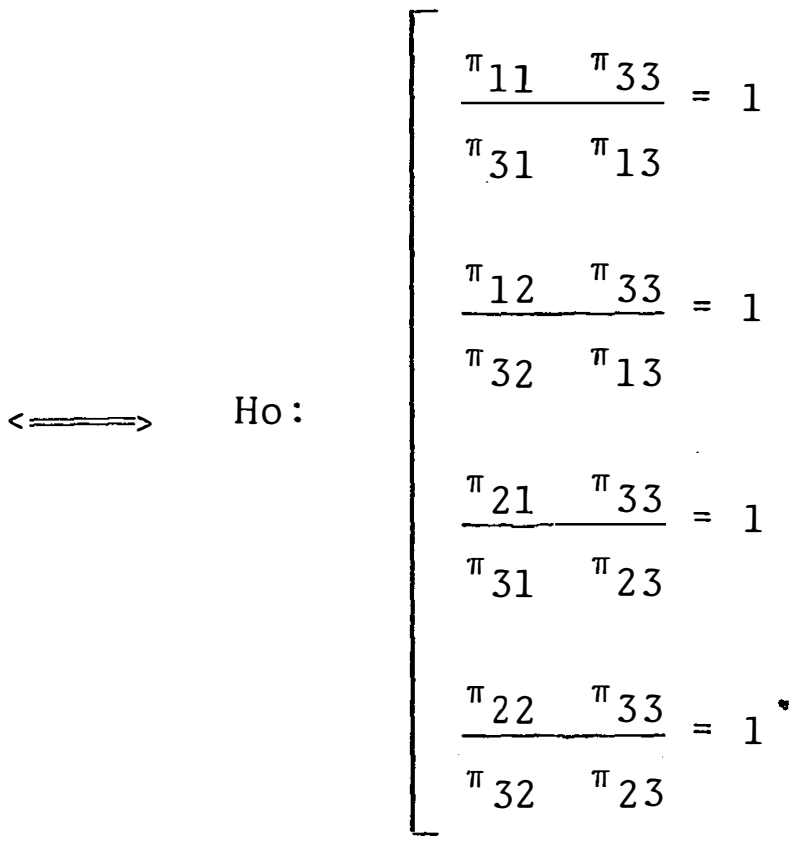

que equivalem a

Ho: $\left[\begin{array}{l}\ln \pi_{11}-\ln \pi_{31}-\ln \pi_{13}+\ln \pi_{33} \\ \ln \pi_{12}-\ln \pi_{32}-\ln \pi_{13}+\ln \pi_{33} \\ \ln \pi_{21}-\ln \pi_{31}-\ln \pi_{23}+\ln \pi_{33} \\ \ln \pi_{22}-\ln \pi_{32}-\ln \pi_{23}+\ln \pi_{33}\end{array}\right.$

que è uma hipōtese do tipo:

Ho: $F(\pi)=K \ln \mathrm{A}_{\sim}^{\pi}=0$ onde

$\mathrm{K}=\left[\begin{array}{rrrrrrrrr}1 & 0 & -1 & 0 & 0 & 0 & -1 & 0 & 1 \\ 0 & 1 & -1 & 0 & 0 & 0 & 0 & -1 & 1 \\ 0 & 0 & 0 & 1 & 0 & -1 & -1 & 0 & 1 \\ 0 & 0 & 0 & 0 & 1 & -1 & 0 & -1 & 1\end{array}\right]$

$A=$ matriz identidade $9 \times 9$ e

$\pi$ é o vetor das proporções 
$\stackrel{\pi}{\prime}^{\prime}=\left[\begin{array}{lllllllll}\pi_{11} & \pi_{12} & \pi_{13} & \pi_{21} & \pi_{22} & \pi_{23} & \pi_{31} & \pi_{32} & \pi_{33}\end{array}\right]$

De um modo geral, numa tabela de contingência c $x$ d a hipótese de independência entre duas respostas é descrita por $(c-1)(d-1)^{\cdot}$ funções do tipo

$F(\pi)=\underset{(c-1)(d-1) x c d}{K} \ln A_{c d x c d} \stackrel{\pi}{\sim} c d x 1$

Essas funções logarítmicas podem ser encontradas com facilidade atravës da medida de associação dada por:

$\begin{aligned} & \Delta_{i j}=\frac{{ }_{i j}{ }^{\pi} \mathrm{cd}}{{ }^{{ }_{c j}{ }^{\pi}{ }_{i d}} \quad \text { com } i}=1,2, \ldots, c-1 e \\ & j=1,2, \ldots, d-1\end{aligned}$

$\Delta_{i j}$ è igual a 1 (um) quando hã independência

Desse modo, podemos substituir a hipótese de independência Ho: $\pi_{i j}=\pi_{i \cdot} \pi_{. j}$ por Ho: $\Delta_{i j}=1$ que se aplicarmos logarítmo, obtemos:

Ho: $\ln \Delta_{i j}=\ln \pi_{i j}-\ln \pi_{c j}-\ln \pi_{i d}+\ln \pi_{c d}=0$

que è da forma

Ho: $F(\pi)=K \ln A \underset{\pi}{\pi}=0$

O teste adequado para essa hipōtese é fornecido por $F^{\prime} S^{-1} F$, que possui distribuição assintōtica de $x^{2}$ com (c-1) (d-1) graus de liberdade, sob Ho.

A hipótese de associação sobre a diagonal prin cipal Ho: $\pi_{i j}=\pi_{i} \pi_{. i}$ para $i=i, 2, \ldots r$ que equivale a uma hipótese do tipo

Ho: $F(\pi)=K \ln A \underset{\sim}{\pi}=0$ 
Vamos desenvolver essa hipótese para $r=4$. Te

mos

$\operatorname{Ho:}\left[\begin{array}{lll}\pi_{11}=\pi_{1} & \pi \cdot 1 \\ \pi_{22}=\pi_{2} & \pi \cdot 2 \\ \pi_{33}=\pi_{3} & \pi \cdot 3 \\ \pi_{44}=\pi_{4} & \pi \cdot 4\end{array}\right.$

Fazendo a transformação logarîtmica, obtemos:

Ho: $\left[\begin{array}{rr}1 \mathrm{n} \pi_{11}-1 \mathrm{n} \pi_{1 .}-1 \mathrm{n} \pi_{.1}=0 \\ 1 \mathrm{n} \pi_{22}-1 \mathrm{n} \pi_{2 .}-1 \mathrm{n} \pi_{.2}=0 \\ 1 \mathrm{n} \pi_{33}-1 \mathrm{n} \pi_{3 .}-1 \mathrm{n} \pi_{.3}=0 \\ 1 \mathrm{n} \pi_{44}-1 \mathrm{n} \pi_{4 .} \quad 1 \mathrm{n} \pi_{.4}=0\end{array}\right.$

que è da forma

Ho: $F(\pi)=K_{4 \times 12}$ in $A_{12 \times 16} \sim \pi 16 \times 1=0$

As matrizes $K, A$ e $\pi$ são as seguintes.

$K=\left[\begin{array}{rrrrrrrrrrrr}1 & 0 & 0 & 0 & -1 & 0 & 0 & 0 & -1 & 0 & 0 & 0 \\ 0 & 1 & 0 & 0 & 0 & -1 & 0 & 0 & 0 & -1 & 0 & 0 \\ 0 & 0 & 1 & 0 & 0 & 0 & -1 & 0 & 0 & 0 & -1 & 0 \\ 0 & 0 & 0 & 1 & 0 & 0 & 0 & -1 & 0 & 0 & 0 & -1\end{array}\right]$ 


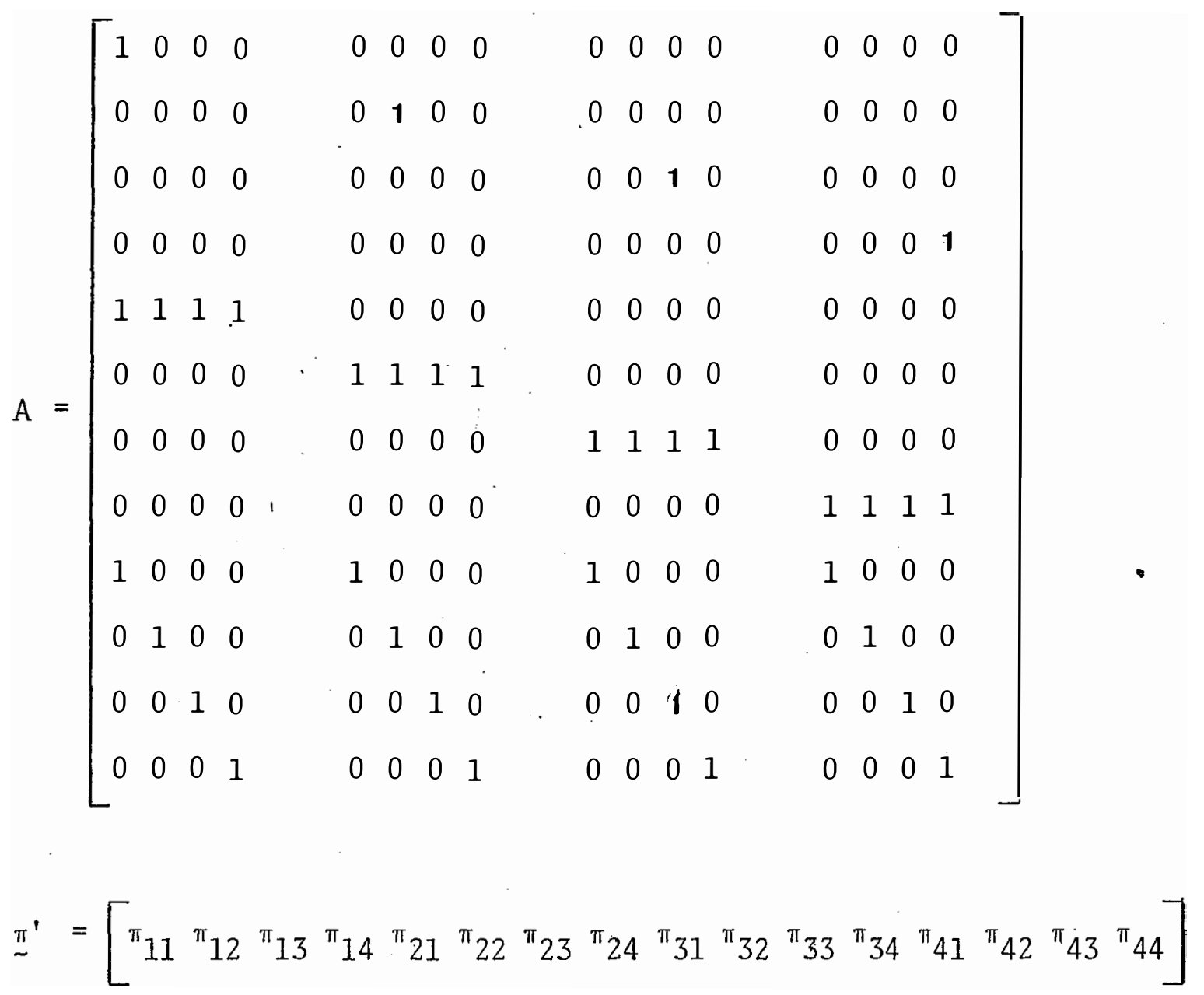

A soma de quadrados para testar esse tipo de hipótese é dada por $F^{\prime} S^{-1} F$ que tem distribuição assintōtica de $x^{2}$ com $r$ graus de liberdade.

Para testarmos hipōteses de independência e as sociação sobre a diagonal principal, que são funções do tipo $F(\pi)=K \ln A \pi$, devemos usar a matriz de variâncias e covariâncias dada por

$\mathrm{S}=\mathrm{K}_{\mathrm{a}}^{-1} \mathrm{AV}(\mathrm{p}) \mathrm{A}^{\prime} \mathrm{D}_{\mathrm{a}}^{-1} \mathrm{~K}^{\prime}$ pois,

$S=H V(p) H^{\prime}$

e H no caso de funções logarítmicas é dada por

$\mathrm{H}=\mathrm{K} \mathrm{D}_{\mathrm{a}}^{-1} \mathrm{~A}$ 
onde $\mathrm{D}_{\mathrm{a}}$ é uma matriz diagonal com elementos do Vetor Ap na diagonal principal.

A hipōtese de homogeneidade das marginais

Ho: $\pi_{i .}=\pi, j$ para $i=j=1,2, \ldots, r$

Para o caso em que $r=4$ podemos escrever

Ho: $\left[\begin{array}{c}\pi_{1}=\pi .1 \\ \pi_{2}=\pi .2 \\ \pi_{3 .}=\pi .3\end{array} \quad \Longrightarrow\right.$ Ho: $\left[\begin{array}{l}\pi_{1 .}-\pi .1=0 \\ \pi_{2-\pi .2}=0 \\ \pi_{3 .}-\pi .3=0\end{array}\right.$

Observação:

$\pi_{4 .}=\pi_{.4} \quad \overrightarrow{\mathrm{e}}$ linearmente dependente.

Podemos reescrever Ho da seguinte forma:

$\mathrm{Ho}:\left[\begin{array}{l}\pi_{11}+\pi_{12}+\pi_{13}+\pi_{14}=\pi_{11}+\pi_{21}+\pi_{31}+\pi_{41} \\ \pi_{21}+\pi_{22}+\pi_{23}+\pi_{24}=\pi_{12}+\pi_{22}+\pi_{32}+\pi_{42} \\ \pi_{31}+\pi_{32}+\pi_{33}+\pi_{34}=\pi_{13}+\pi_{23}+\pi_{33}+\pi_{43}\end{array}\right]$

ou ainda

$\mathrm{Ho}:\left[\begin{array}{l}\pi_{12}+\pi_{13}+\pi_{14}-\pi_{21}-\pi_{31}-\pi_{41}=0 \\ \pi_{21}+\pi_{23}+\pi_{24}-\pi_{12}-\pi_{32}-\pi_{42}=0 \\ \pi_{31}+\pi_{32}+\pi_{34}-\pi_{13}-\pi_{23}-\pi_{43}=0\end{array}\right]$

o que implica em

Ho: $\left[\begin{array}{ll}F_{1} & (\pi) \\ F_{2} & (\pi) \\ F_{3} & (\pi)\end{array}\right]=0$ ou $\underset{\sim}{F}(\pi)=0$ 
onde as funções são do tipo

$F(\pi)=A \underline{\pi}$

As matrizes A e $\underset{\sim}{\pi}$ são as seguintes

$\stackrel{A}{3}_{3 \times 16}=\left[\begin{array}{rrrrrrrrrrrrrrrr}0 & 1 & 1 & 1 & 1-1 & 0 & 0 & 0 & -1 & 0 & 0 & 0 & -1 & 0 & 0 & 0 \\ 0 & -1 & 0 & 0 & 1 & 0 & 1 & 1 & 0 & -1 & 0 & 0 & 0 & -1 & 0 & 0 \\ 0 & 0 & -1 & 0 & 0 & 0 & -1 & 0 & 1 & 1 & 0 & 1 & 0 & 0 & -1 & 0\end{array}\right]$

$\underline{\sim}^{\prime}=\left[\begin{array}{llllllllllllllll}\pi_{11} & \pi_{12} & \pi_{13} & \pi_{14} & \pi_{21} & \pi_{22} & \pi_{23} & \pi_{24} & \pi_{31} & \pi_{32} & \pi_{33} & \pi_{34} & \pi_{41} & \pi_{42} & \pi_{43} & \pi_{44}\end{array}\right]$

0 teste adequado é dado por

$\mathrm{SQ}[\mathrm{F}(\pi)=0]=\mathrm{F}^{\prime} \mathrm{S}^{-1} \mathrm{~F}=(\mathrm{Ap})^{\prime} \mathrm{S}^{-1} \mathrm{Ap}$

que tem distribuição assintótica de $x^{2}$ com $r-1$ grau de liberdade, sob Ho.

A hipótese de simetria

Ho: $\pi_{i j}=\pi_{j i}$ com $i \neq j$ e $i, j=1,2, \ldots, r$

Para o caso de $r=4$ temos

Ho: $\left[\begin{array}{l}\pi_{12}=\pi_{21} \\ \pi_{13}=\pi_{31} \\ \pi_{14}=\pi_{41} \\ \pi_{23}=\pi_{32} \\ \pi_{24}=\pi_{42} \\ \pi_{34}=\pi_{43}\end{array} \quad\right.$ Ho: $\left[\begin{array}{l}\pi_{12}-\pi_{21}=0 \\ \pi_{13}-\pi_{31}=0 \\ \pi_{14}-\pi_{41}=0 \\ \pi_{23}-\pi_{32}=0 \\ \pi_{24}-\pi_{42}=0 \\ \pi_{34}-\pi_{43}=0\end{array}\right.$

que é do tipo Ho: $F(\pi)=A=0$, onde 
$A_{6 \times 16}=\left[\begin{array}{rrrrrrrrrrrrrrrr}0 & 1 & 0 & 0 & -1 & 0 & 0 & 0 & 0 & 0 & 0 & 0 & 0 & 0 & 0 & 0 \\ 0 & 0 & 1 & 0 & 0 & 0 & 0 & 0 & -1 & 0 & 0 & 0 & 0 & 0 & 0 & 0 \\ 0 & 0 & 0 & 1 & 0 & 0 & 0 & 0 & 0 & 0 & 0 & 0 & -1 & 0 & 0 & 0 \\ 0 & 0 & 0 & 0 & 0 & 0 & 1 & 0 & 0 & -1 & 0 & 0 & 0 & 0 & 0 & 0 \\ 0 & 0 & 0 & 0 & 0 & 0 & 0 & 1 & 0 & 0 & 0 & 0 & 0 & -1 & 0 & 0 \\ 0 & 0 & 0 & 0 & 0 & 0 & 0 & 0 & 0 & 0 & 0 & 1 & 0 & 0 & -1 & 0\end{array}\right]$

e

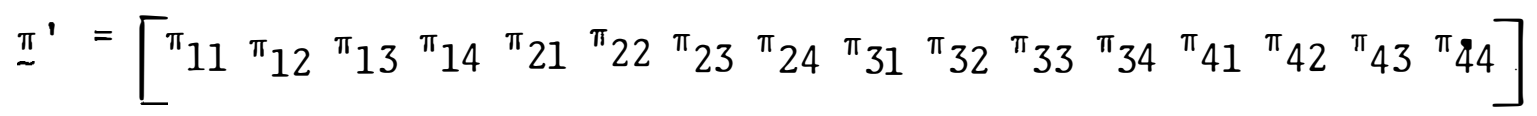

o teste estatístico adequado a essa hipótese é

dado por:

$\mathrm{SQ}[\mathrm{F}(\pi)=0]=\mathrm{F}^{\prime} \mathrm{S}^{-1} \mathrm{~F}=(\mathrm{Ap}) \cdot \mathrm{S}^{-1}$ (Ap)

que tem distribuição assintótica de $x^{2} \operatorname{com} \frac{r(r-1)}{2}$ graus de $1 \underline{i}$ berdade, sob Ho.

o segundo tipo de tabela de contingência mais conhecido é a tabela 1 apresentada na página 11, na qual, é baseado todo o método de GRIZZLE et alii.

Para esse tipo de tabela, as hipóteses podem ser ainda mais diversificada, pois, podemos adaptar a elas mo delos matemáticos convenientes a cada experimento. Um desses modelos é adaptado ao experimento odontológico, desenvolvido no próximo item. 
3.3. Aplicação do Método "GSK" em um Experimento Odontológico.

3.3.1. O Experimento

Após o tratamento de canais radiculares, faz-se radiografias do dente tratado e observa-se a reatividade (capacidade de reagir ou não a tratamento químico).

Cinco fatores são incluídos nestes experimento e para cada fator consideramos dois níveis que são os seguintes:

\begin{tabular}{|c|c|c|}
\hline FATORES & $N \quad I V E$ & I $S$ \\
\hline I DADE & Menos que 30 anos & 30 anos ou mais \\
\hline TRATAMENTO & Parcial & Total \\
\hline SEXO & Masculino & Feminino \\
\hline MAXILAR & superior & Inferior \\
\hline$N \cong$ DE RAIZES & uma raiz & mais que uma raiz \\
\hline
\end{tabular}


Assim temos para o referido experimento $32\left(2^{5}\right)$ sub-populaçōes binomiais pois a variāvel de anālise (reatividade) é composta apenas de duas categorias de respostas (com reação ou sem reação) .

\subsubsection{Metodologia}

Analisaremos o experimento através de um dèlineamento fatorial pois assim, poderemos testar se as proporções de respostas "com reação" são equivalentes para todos os fatores e todas as suas interações.

Para isto adotaremos o modelo matemätico completo que segue.

$$
\begin{aligned}
Y & =\mu+\alpha_{1} x_{1}+\alpha_{2} x_{2}+\alpha_{3} x_{3}+\alpha_{4} x_{4}+\alpha_{5} x_{5}+\beta_{1} x_{1} x_{2}+ \\
& +\beta_{2} x_{1} x_{3}+\beta_{3} x_{1} x_{1}+\beta_{4} x_{1} x_{5}+\beta_{5} x_{2} x_{3}+\beta_{6} x_{2} x_{4}+\beta_{7} x_{2} x_{5}+ \\
& +\beta_{8} x_{3} x_{4}+\beta_{9} x_{3} x_{5}+\beta_{10} x_{4} x_{5}+\gamma_{1} x_{1} x_{2} x_{3}+\gamma_{2} x_{1} x_{2} x_{4}+ \\
& +\gamma_{3} x_{1} x_{2} x_{5}+\gamma_{4} x_{1} x_{3} x_{4}+\gamma_{5} x_{1} x_{3} x_{5}+\gamma_{6} x_{1} x_{4} x_{5}+ \\
& +\gamma_{7} x_{2} x_{3} x_{4}+\gamma_{8} x_{2} x_{3} x_{5}+\gamma_{9} x_{2} x_{4} x_{5}+\gamma_{10} x_{3} x_{4} x_{5}+ \\
& +\psi_{1} x_{1} x_{2} x_{3} x_{4}+\psi_{2} x_{1} x_{2} x_{3} x_{5}+\psi_{3} x_{1} x_{2} x_{4} x_{5}+\psi_{4} x_{1} x_{3} x_{4} x_{5}+ \\
& +\psi_{5} x_{2} x_{3} x_{4} x_{5}+\Gamma_{1} x_{1} x_{2} x_{3} x_{4} x_{5}+\ell_{1}
\end{aligned}
$$

Encontrada a regressão, segundo o método"GSK", onde, $b=\left(X^{\prime} S^{-1} X\right)^{-1} X S^{-1} F$ é a estimativa da matriz de parâmetros, testaremos os parâmetros, através do teste esta- 
tístico.

$Q C=S Q\{c \beta=0\}=(c b)^{\prime}\left\{c\left(X, \quad S^{-1} X\right)^{-1} c\right\}^{-1}(c b)$

Verifica-se a significiância ou não dos parâme tros, os parâmetros não significantes são eliminados para obtermos, com ist.o, alguns graus de liberdade para testarmos o ajustamento do modelo através do teste.

$S Q\{F(\pi)=.X \beta\}=F^{\prime} \quad S^{-1} F-b^{\prime}\left\{X \quad S^{-1} X\right\} \quad b$

Os dados, para este experimento, foram obtidos junto ao departamento de Diagnóstico e Cirurgia, da Faculdade de Odontologia de Araraquara.

A seguir apresentamos os dados já tabulados nas diversas sub-populações. 
TABELA 4: DADOS DE REATIVIDADE SEGUNDO AS SUB-POPULAÇOES

\begin{tabular}{|c|c|c|c|c|c|c|c|}
\hline $\begin{array}{l}\text { IDADE } \\
\text { (ANOS) }\end{array}$ & TRATAMENTO & SEXO & MAXILAR & No & RAIZES & COM REAÇÃO & SEM REAÇÃO \\
\hline$<30$ & PARCIAL & M & SUP & & 1 & 49 & 15 \\
\hline$<30$ & PARC IAL & M & SUP & & $>1$ & 24 & 16 \\
\hline$<30$ & PARCIAL & M & INF & & 1 & 05 & 03 \\
\hline$<30$ & PARCIAL & M & INF & & $>1$ & 38 & 15 \\
\hline$<30$ & PARCIAL & F & SUP & & 1 & 182 & 49 \\
\hline$<30$ & PARCIAL & F & SUP & & $>1$ & 99 & 45 \\
\hline$<30$ & PARCIAL & F & INF & & 1 & 17 & 01 \\
\hline$<30$ & PARCIAL & $\mathrm{F}$ & INF & & $>1$ & 78 & 27. \\
\hline$<30$ & TOTAL & M & SUPP & & 1 & 55 & 37 \\
\hline$<30$ & TOTAL & M & SUP & & $>1$ & 23 & 12 \\
\hline$<30$ & TOTAL & M & INF & & 1 & 02 & 01 \\
\hline$<30$ & TOTAL & M & INF & & $>1$ & 21 & 08 \\
\hline$<30$ & TOTAL & F & SUP & & 1 & 139 & 73 \\
\hline$<30$ & TOTAL & $\mathrm{F}$ & SUP & & $>1$ & 51 & 41 \\
\hline$<30$ & TOTAL & $\mathrm{F}$ & INF & & 1 & 05 & 08 \\
\hline$<30$ & TOTAL & F & INF & & $>1$ & 38 & 30 \\
\hline$\geq 30$ & PARCIAL & M & SUP & & 1 & 35 & 16 \\
\hline$\geq 30$ & PARCIAL & M & SUP & & $>1$ & 30 & 13 \\
\hline$\geq 30$ & PARCIAL & M & INF & & 1 & 11 & 07 \\
\hline$\geq 30$ & PARCIAL & M & INF & & $>1$ & 18 & 09 \\
\hline$\geq 30$ & PARCIAL & F & SUP & & $\cdot 1$ & 218 & 83 \\
\hline$\geq 30$ & PARCIAL & $F$ & SUP & & $>1$ & 135 & 62 \\
\hline$\geq 30$ & PARCIAL & F & INF & & . 1 & 58 & 24 \\
\hline$\geq 30$ & PARCIAL & $\mathrm{F}$ & INF & & $>1$ & 87 & 56 \\
\hline$\geq 30$ & TOTAL & M & SUP & & 1 & 19 & 16 \\
\hline$\geq 30$ & TOTAL & M & SUP & & $>1$ & 06 & 09 \\
\hline$\geq 30$ & TOTAL & M & INF & & 1 & 06 & 06 \\
\hline$\geq 30$ & TOTAL & M & INF & & $>1$ & 10 & 07 \\
\hline$\geq 30$ & TOTAL & F & SUP & & 1 & 92 & 68 \\
\hline$\geq 30$ & TOTAL & $F$ & SUP & & $>1$ & 40 . & 44 \\
\hline$\geq 30$ & TOTAL & F & INF & & 1 & 50 & 15 \\
\hline$\geq 30$ & TOTAL & F & INF & & $>1$ & 40 & 32 \\
\hline
\end{tabular}




\subsubsection{Anālise do experimento pelo modelo GSK}

A partir desta distribuição de frequência pelas sub-populações e do modelo adotado temos:

3.3.3.1. Matriz das proporções observadas

$$
\hat{\pi}=\underline{p}=\left[\begin{array}{c}
49 / 64 \\
15 / 64 \\
24 / 40 \\
16 / 40 \\
\cdot \cdot \\
\cdot \cdot \\
40 / 72 \\
32 / 72
\end{array}\right]_{64 \times 1}
$$

3.3.3.2. Matriz de variâncias e covariâncias

Para cada sub-população teremos uma matrĩz 2X2 de Variâncias - covariâncias do tipo

$$
\underline{V}\left(p_{i}\right)=\frac{1}{n_{i}}\left[\begin{array}{cc}
p_{i 1}\left(1-p_{i 1}\right) & -p_{i 1} p_{i 2} \\
-p_{i 1} p_{i 2} & p_{i 2}\left(1-p_{i 2}\right)
\end{array}\right]
$$

em se tratando de variāel binomial temos $\left(1-p_{i 1}\right)=p_{i 2}$ e então

$$
\underline{\mathrm{V}}\left(\mathrm{p}_{\mathrm{i}}\right)=\frac{1}{\mathrm{n}_{\mathrm{i}}}\left[\begin{array}{cc}
\mathrm{p}_{\mathrm{i}} \mathrm{p}_{\mathrm{i} 2} & -\mathrm{p}_{\mathrm{il}} \mathrm{p}_{\mathrm{i} 2} \\
-\mathrm{p}_{i 1} \mathrm{p}_{\mathrm{i} 2} & \mathrm{p}_{\mathrm{il}} \mathrm{p}_{\mathrm{i} 2}
\end{array}\right]
$$




$$
\begin{aligned}
& \text { Para } i=1,2,3, \ldots, 32 \text { temos: } \\
& \underline{\mathrm{V}}\left(\mathrm{p}_{1}\right)=\left[\begin{array}{rr}
0,002804 & -0,002804 \\
-0,002804 & 0,002804
\end{array}\right] \underset{\mathrm{V}}{\mathrm{V}}\left(\mathrm{p}_{2}\right)=\left[\begin{array}{rr}
0,006000 & -0,006000 \\
-0,006000 & 0,006000
\end{array}\right] \\
& \underline{\mathrm{V}}\left(\mathrm{p}_{3}\right)=\left[\begin{array}{rr}
0,029297 & -0,029297 \\
-0,029297 & 0,029297
\end{array}\right] \underset{\mathrm{V}}{\sim}\left(\mathrm{p}_{4}\right)=\left[\begin{array}{rr}
0,003829 & -0,003839 \\
-0,003829 & 0,003829
\end{array}\right] \\
& \underline{\mathrm{V}}\left(\mathrm{p}_{5}\right)=\left[\begin{array}{rr}
0,000723 & -0,000723 \\
-0,000723 & 0,000723
\end{array}\right] \mathrm{V}\left(\mathrm{p}_{6}\right)=\left[\begin{array}{rr}
0,001492 & -0,001492 \\
-0,001492 & 0,001492
\end{array}\right] \\
& \underline{\mathrm{V}}\left(\mathrm{p}_{7}\right)=\left[\begin{array}{rr}
0,002915 & -0,002915 \\
-0,002915 & 0,002915
\end{array}\right] \underline{\mathrm{V}}\left(\mathrm{p}_{8}\right)=\left[\begin{array}{rr}
0,001819 & -0,001819 \\
-0,001819 & 0,001819
\end{array}\right] \\
& \underset{\sim}{\mathrm{V}}\left(\mathrm{p}_{9}\right)=\left[\begin{array}{rr}
0,002613 & -0,002613 \\
-0,002613 & 0,002613
\end{array}\right] \underset{\mathrm{V}}{\sim}\left(\mathrm{p}_{10}\right)=\left[\begin{array}{rr}
0,006437 & -0,006437 \\
-0,006437 & 0,006437
\end{array}\right] \\
& \underline{\mathrm{V}}\left(\mathrm{p}_{11}\right)=\left[\begin{array}{rr}
0,074074 & -0,074074 \\
-0,074074 & 0,074074
\end{array}\right] \underline{\mathrm{V}}\left(\mathrm{p}_{12}\right)=\left[\begin{array}{rr}
0,006888 & -0,006888 \\
-0,006888 & 0,006888
\end{array}\right] \\
& \underline{\mathrm{V}}\left(\mathrm{p}_{13}\right)=\left[\begin{array}{rr}
0,001065 & -0,001065 \\
-0,001065 & 0,001065
\end{array}\right] \underset{\mathrm{V}}{ }\left(\mathrm{p}_{14}\right)=\left[\begin{array}{rr}
0,002685 & -0,002685 \\
-0,002685 & 0,002685
\end{array}\right] \\
& \underset{\sim}{V}\left(\mathrm{p}_{15}\right)=\left[\begin{array}{rr}
0,018207 & -0,018207 \\
-0,018207 & 0,018207
\end{array}\right] \underset{\mathrm{V}}{ }\left(\mathrm{p}_{16}\right)=\left[\begin{array}{rr}
0,003626 & -0,003626 \\
-0,003626 & 0,003626
\end{array}\right] \\
& \underset{\sim}{V}\left(\mathrm{p}_{17}\right)=\left[\begin{array}{rr}
0,004222 & -0,004222 \\
-0,004222 & 0,004222
\end{array}\right] \underset{\mathrm{V}}{ }\left(\mathrm{p}_{18}\right)=\left[\begin{array}{rr}
0,004905 & -0,004905 \\
-0,004905 & 0,004905
\end{array}\right]
\end{aligned}
$$




$$
\begin{aligned}
& \mathrm{V}\left(\mathrm{p}_{19}\right)=\left[\begin{array}{rr}
0,013203 & -0,013203 \\
-0,013203 & 0,013203
\end{array}\right] \underline{\mathrm{V}}\left(\mathrm{p}_{20}\right)=\left[\begin{array}{rr}
0,008230 & -0,008230 \\
-0,008230 & 0,008230
\end{array}\right] \\
& \underset{\sim}{V}\left(p_{21}\right)=\left[\begin{array}{rr}
0,000663 & -0,000663 \\
-0,000663 & 0,000663
\end{array}\right] \underset{\sim}{V}\left(p_{22}\right)=\left[\begin{array}{rr}
0,001095 & -0,001095 \\
-0,001095 & 0,001095
\end{array}\right] \\
& \underline{v}\left(p_{23}\right)=\left[\begin{array}{rr}
0,002525 & -0,002525 \\
-0,002525 & 0,002525
\end{array}\right] \underline{V}\left(p_{24}\right)\left[\begin{array}{cc}
0,001666 & -0,001666 \\
-0,001666 & 0,001666
\end{array}\right] \\
& \underset{\sim}{\mathrm{V}}\left(\mathrm{p}_{25}\right)=\left[\begin{array}{rr}
0,007090 & -0,007090 \\
-0,007090 & 0,007090
\end{array}\right] \underset{\sim}{\mathrm{V}}\left(\mathrm{p}_{26}\right)\left[\begin{array}{rr}
0,016000 & -0,16000 \\
-0,016000 & 0,16000
\end{array}\right] \\
& \underset{\sim}{\mathrm{V}}\left(\mathrm{p}_{27}\right)=\left[\begin{array}{rr}
0,020833 & -0,020833 \\
-0,020833 & 0,020833
\end{array}\right] \underset{\sim}{\mathrm{V}}\left(\mathrm{p}_{28}\right)=\left[\begin{array}{rr}
0,014248 & -0,014248 \\
-0,014248 & 0,014248
\end{array}\right] \\
& \underset{\sim}{\mathrm{V}}\left(\mathrm{p}_{29}\right)=\left[\begin{array}{rr}
0,001527 & -0,001527 \\
-0,001527 & 0,001527
\end{array}\right] \underset{\sim}{\mathrm{V}}\left(\mathrm{p}_{30}\right)=\left[\begin{array}{rr}
0,002969 & -0,002969 \\
-0,002969 & 0,002969
\end{array}\right] \\
& \underset{\sim}{V}\left(p_{31}\right)=\left[\begin{array}{rr}
0,002731 & -0,002731 \\
-0,002731 & 0,002731
\end{array}\right] \underset{\sim}{V}\left(p_{32}\right)=\left[\begin{array}{rr}
0,003429 & -0,003429 \\
-0,003429 & 0,003429
\end{array}\right]
\end{aligned}
$$

A matriz completa de variânciase covariâncias é uma matriz bloco diagonal pois as sub-populações são independentes, o que nos dá covariância nula entre as populações, por tanto teremos $V(p)$ igual a: 


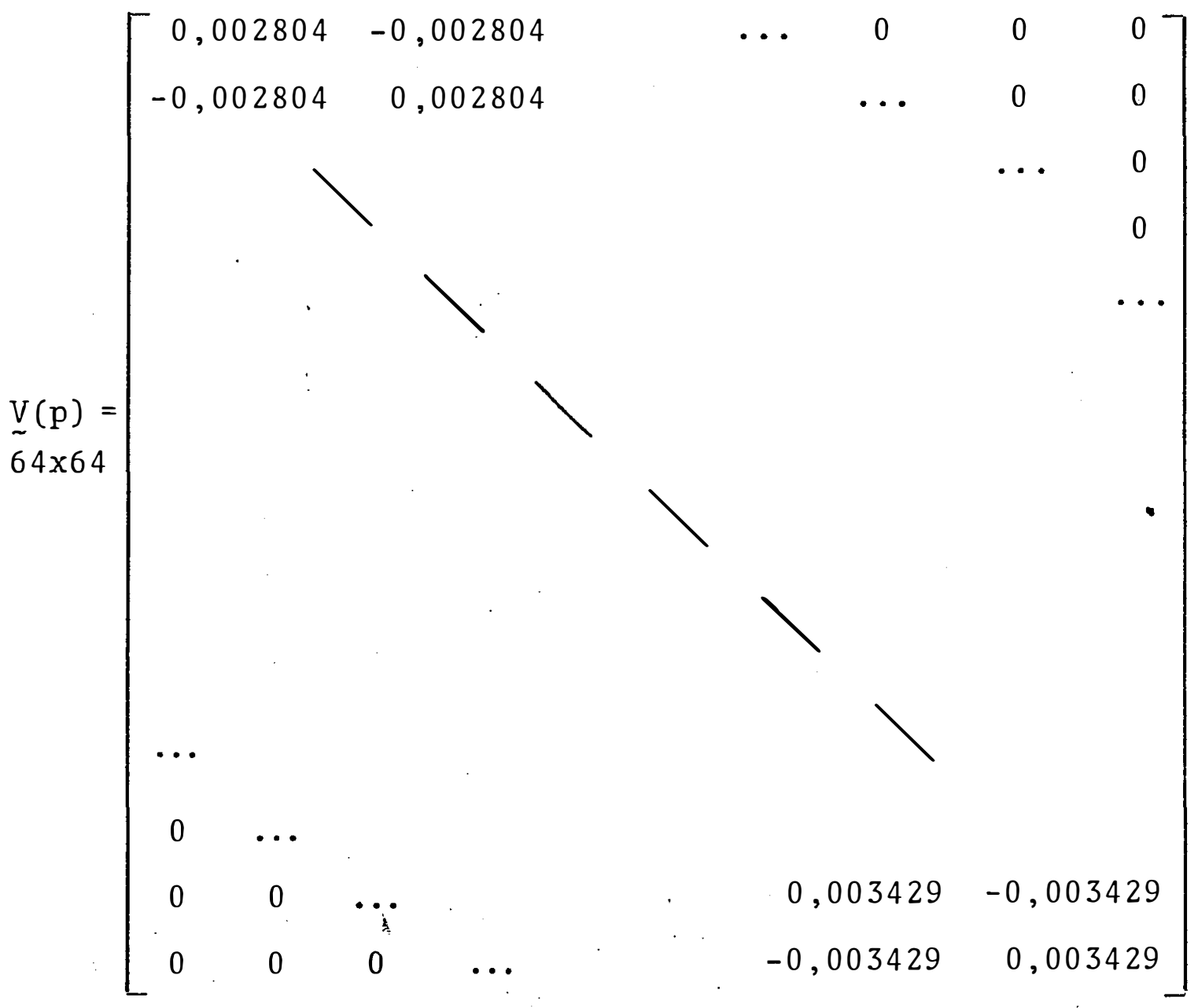

$3 \cdot 3 \cdot 3 \cdot 3$. Matriz $\stackrel{A}{\sim}$

Como estamos interessados em comparar as proporções obtidas com a resposta "com reação" podemos construir uma matriz de 32 funções $F m(p)$ tal que suas derivadas parciais em relação a $p_{i j}$ nos dêem seguinte matriz $\underset{\sim}{A}$ 


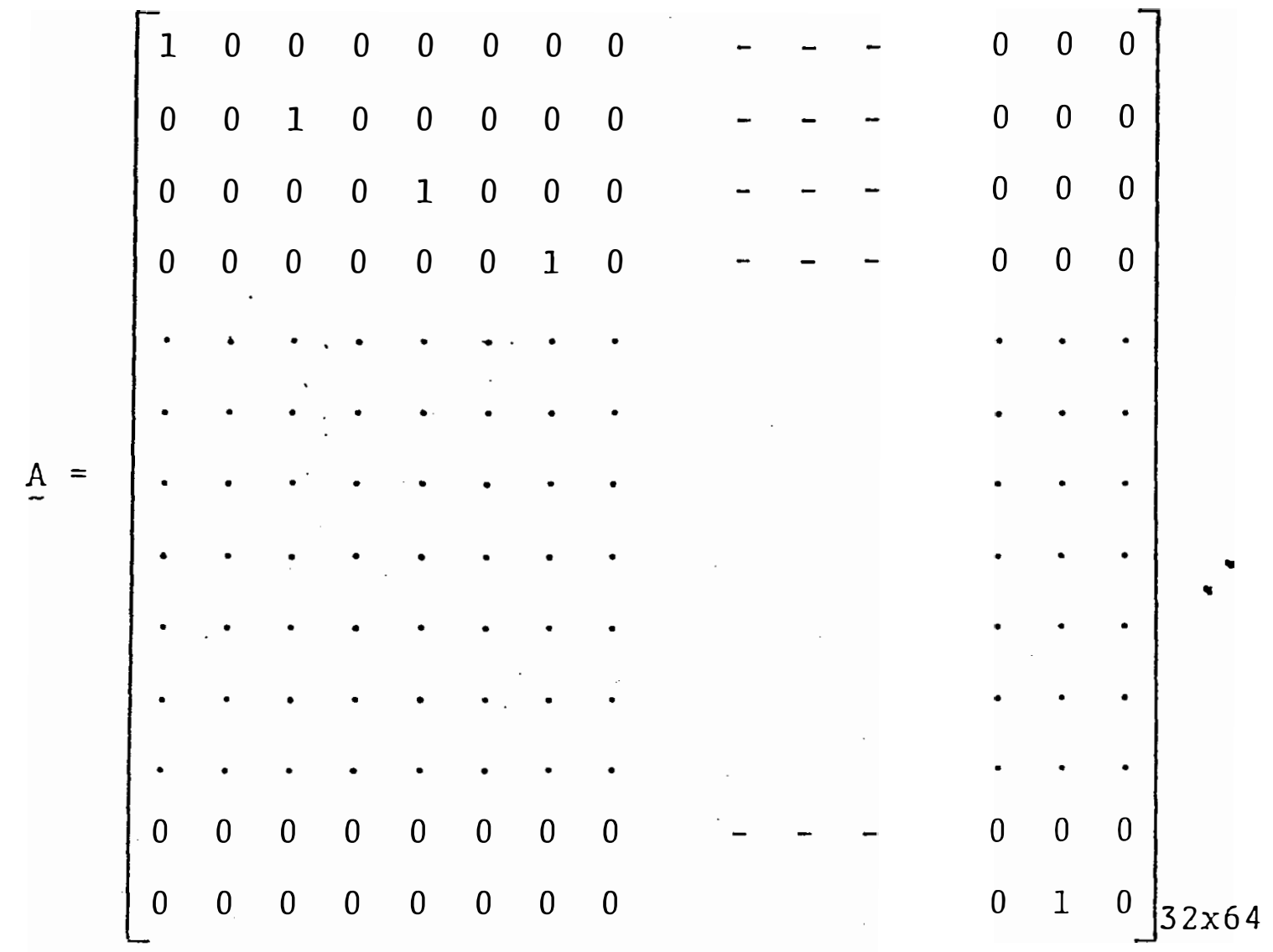

\subsubsection{Matriz $\underset{-}{X}$}

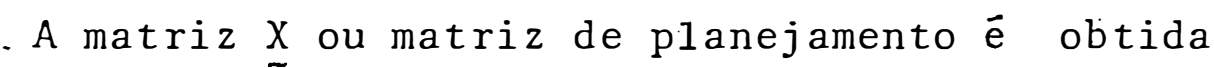
através da seguinte estruturação dos fatores principais:

Idade 1 se o paciente tiver menos que 30 anos - I se o paciente tiver 30 anos ou mais

Tratamento

1 se o tratamento foi parcial -1 se o tratamento foi total

Sexo

1 se o paciente foi do sexo masculino

-1 se o paciente for do sexo feminino 
Maxilar

1 se o dente tratado estiver no maxilar superior - 1 se o dente tratado estiver no maxilar inferior

\author{
1 se o dente tratado tem uma raiz \\ $n \circ$ de raizes \\ - 1 se o dente tratado tem mais de uma raiz
}

Portanto a matriz $\mathrm{X}$ de planejamento é construí da da seguinte forma.

A primeira coluna é toda de números 1 , poiss, re rere-se à média do modelo. As cinco colunas que seguem (da 2 a a $6^{\mathrm{a}}$ ), , referem-se aos fatores principais, e seguem a estruturação acima descrita, assumindo valores 1 e -1 , dependendo do fator e do nível do fator considerado, seguindo sempre a ordem de disposição da tabela (4). Da $7^{\mathrm{a}}$. a $32^{\mathrm{a}}$. colunas, relativas às interações dos fatores principais, são obtidas através de multiplicações das colunas dos efeitos principais envolvidos em cada interação.

Portanto a matriz $\mathrm{X}$ fica: 


$$
\begin{aligned}
& \begin{array}{llllllll}
\mu & \alpha_{1} & \alpha_{2} & \alpha_{3} & \alpha_{4} & \alpha_{5} & \beta_{1} & \gamma_{1}
\end{array} \\
& {\left[\begin{array}{lllllllllllll}
1 & 1 & 1 & 1 & 1 & 1 & 1 & \ldots & 1 & \ldots & 1 & \ldots & 1
\end{array}\right]} \\
& \begin{array}{lllllllllllll}
1 & 1 & 1 & 1 & 1 & -1 & 1 & \ldots & 1 & \ldots & 1 & \ldots & -1
\end{array} \\
& \begin{array}{lllllllllllll}
1 & 1 & 1 & 1 & -1 & 1 & 1 & \ldots & 1 & \ldots & -1 & \ldots & -1
\end{array} \\
& \begin{array}{llllllllllllll}
1 & 1 & 1 & 1 & -1 & -1 & 1 & \ldots & 1 & \ldots & -1 & \ldots & 1
\end{array}
\end{aligned}
$$

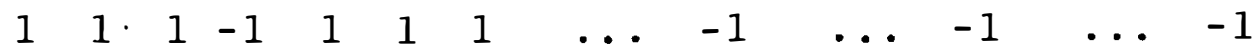

$$
\begin{aligned}
& \begin{array}{lllllllllllllll}
1 & 1 & 1 & -1 & 1 & -1 & 1 & \ldots & -1 & \ldots & -1 & \ldots & 1
\end{array} \\
& \begin{array}{lllllllllllll}
1 & 1 & 1 & -1 & -1 & 1 & 1 & \ldots & -1 & \ldots & 1 & \ldots & 1
\end{array} \\
& \begin{array}{llllllllllllll}
1 & 1 & 1 & -1 & -1 & -1 & 1 & \ldots & -1 & \ldots & 1 & \ldots & -1
\end{array} \\
& \begin{array}{llllllllllllll}
1 & 1 & -1 & 1 & 1 & 1 & -1 & \ldots & -1 & \ldots & -1 & \ldots & -1
\end{array} \\
& \begin{array}{llllllllllllll}
1 & 1 & -1 & 1 & 1 & -1 & -1 & \ldots & -1 & \ldots & -1 & \ldots & 1
\end{array} \\
& \begin{array}{lllllllllllll}
1 & 1 & -1 & 1 & -1 & 1 & -1 & \ldots & -1 & \ldots & 1 & \ldots & 1
\end{array} \\
& \begin{array}{llllllllllllll}
1 & 1 & -1 & 1 & -1 & -1 & -1 & \ldots & -1 & \ldots & 1 & \ldots & -1
\end{array} \\
& \begin{array}{lllllllllllll}
1 & 1 & -1 & -1 & 1 & 1 & -1 & \ldots & 1 & \ldots & 1 & \ldots & -1
\end{array} \\
& \begin{array}{lllllllllllll}
1 & 1 & -1 & -1 & 1 & -1 & -1 & \ldots & 1 & \ldots & 1 & \ldots & 1
\end{array} \\
& \begin{array}{lllllllllllll}
1 & 1 & -1 & -1 & -1 & 1 & -1 & \ldots & 1 & \ldots & -1 & \ldots & -1
\end{array}
\end{aligned}
$$

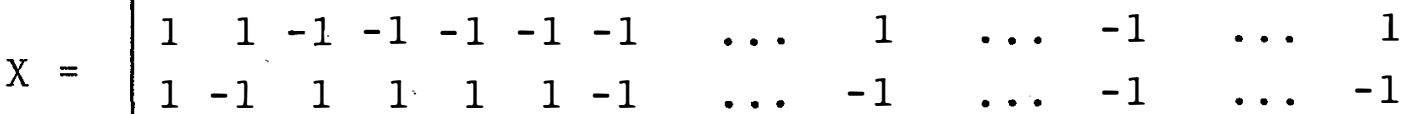

$$
\begin{aligned}
& \begin{array}{llllllllllllll}
1 & -1 & 1 & 1 & 1 & 1 & -1 & \ldots & -1 & \ldots & -1 & \ldots & -1
\end{array} \\
& \begin{array}{lllllllllllllll}
1 & -1 & 1 & 1 & 1 & -1 & -1 & \ldots & -1 & \ldots & -1 & \ldots & 1
\end{array} \\
& \begin{array}{llllllllllllll}
1 & -1 & 1 & 1 & -1 & 1 & -1 & \ldots & -1 & \ldots & 1 & \ldots & 1
\end{array} \\
& \begin{array}{llllllllllllll}
1 & -1 & 1 & 1 & -1 & -1 & -1 & \ldots & -1 & \ldots & 1 & \ldots & -1
\end{array} \\
& \begin{array}{llllllllllllll}
1 & -1 & 1 & -1 & 1 & 1 & -1 & \ldots & 1 & \ldots & -1 & \ldots & 1
\end{array} \\
& \begin{array}{lllllllllllll}
1 & -1 & 1 & -1 & 1 & -1 & -1 & \ldots & 1 & \ldots & -1 & \ldots & -1
\end{array} \\
& \begin{array}{llllllllllllll}
1 & -1 & 1 & -1 & -1 & 1 & -1 & \ldots & 1 & \ldots & -1 & \ldots & -1
\end{array} \\
& \begin{array}{llllllllllllll}
1 & -1 & 1 & -1 & -1 & -1 & -1 & \ldots & 1 & \ldots & -1 & \ldots & 1
\end{array} \\
& \begin{array}{lllllllllllll}
1 & -1 & -1 & 1 & 1 & 1 & 1 & \ldots & 1 & \ldots & 1 & \ldots & 1
\end{array} \\
& \begin{array}{lllllllllllll}
1 & -1 & -1 & 1 & 1 & -1 & 1 & \ldots & 1 & \ldots & 1 & \ldots & -1
\end{array} \\
& \begin{array}{lllllllllllll}
1 & -1 & -1 & 1 & -1 & 1 & 1 & \ldots & 1 & \ldots & -1 & \ldots & -1
\end{array} \\
& \begin{array}{lllllllllllll}
1 & -1 & -1 & 1 & -1 & -1 & 1 & \ldots & 1 & \ldots & -1 & \ldots & 1
\end{array} \\
& \begin{array}{lllllllllllll}
1 & -1 & -1 & -1 & 1 & 1 & 1 & \ldots & -1 & \ldots & -1 & \ldots & -1
\end{array} \\
& \begin{array}{lllllllllllll}
1 & -1 & -1 & -1 & 1 & -1 & 1 & \ldots & -1 & \ldots & -1 & \ldots & 1
\end{array} \\
& \begin{array}{lllllllllllll}
1 & -1 & -1 & -1 & -1 & 1 & 1 & \ldots & -1 & \ldots & 1 & \ldots & 1
\end{array} \\
& \left.\begin{array}{llllllllllllll}
1 & -1 & -1 & -1 & -1 & -1 & 1 & \ldots & -1 & \ldots & 1 & \ldots & -1
\end{array}\right] 2 \times 32
\end{aligned}
$$


As letras gregas colocadas fora da matriz $\mathrm{X}$, acima de cada coluna, são os parâmetros aos quais as colunas se referem e estão apenas auxiliando a montagem da matriz.

A média do modelo é representada por $\mu, \alpha_{1}$ é o parâmetro relativo à idade, $\alpha_{2}$ parâmetro relativo ao tratamen to, $\alpha_{3}$ parâmetro relativo ao sexo, $\alpha_{4}$ parâmetro relativo ao maxilar e $\alpha_{5}$ o parâmetro relativo ao $n^{\circ}$ de raízes do dente.

Como exemplo construímos ainda as colunas, $\beta_{1}$ (Interação Idade $x$ Tratamento) obtida pelo produto da 2 a è 3 . colunas, $\gamma_{1}$ (Interação Idade $X$ Tratamento $X$ Sexo) obtida pelo

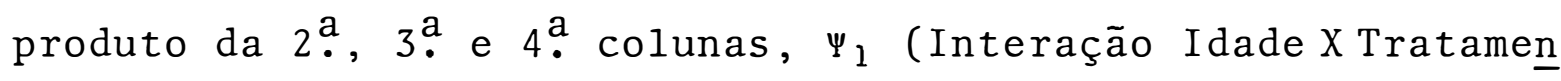

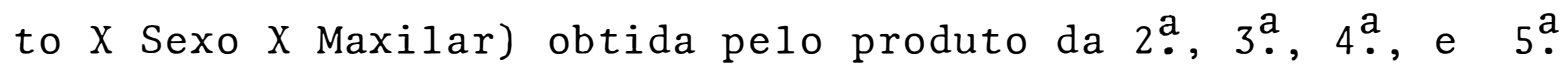
colunas, $\tau_{1}$ (Interação de todos os fatores principais) obtida pelo produto da $2^{\mathrm{a}} \cdot, 3 \stackrel{\mathrm{a}}{\cdot}, 4 \stackrel{\mathrm{a}}{\cdot}, 5^{\mathrm{a}}$. e $6^{\mathrm{a}} \cdot$ colunas.

\subsubsection{Matriz $\mathrm{S}^{-1}$}

A matriz de Variâncias-covariâncias. de $\underset{\sim}{F}$ é dada pela förmula

$$
\begin{aligned}
& \mathrm{S}=\mathrm{A} \quad \mathrm{V}(\mathrm{p}) \quad \mathrm{A}^{\prime} \\
& 32 \times 32 \quad 32 \times 64 \quad 64 \times 64 \quad 64 \times 32 \\
& \text { Portanto, S è uma matriz diagonal. }
\end{aligned}
$$




$$
\begin{aligned}
S= & \text { diagonal }[0,002804 ; 0,006000 ; 0,029297 ; 0,003829 ; \\
& 0,000723 ; 0,001492 ; 0,002915 ; 0,001819 ; 0,002613 ; \\
& 0,006437 ; 0,074074 ; 0,006888 ; 0,001065 ; 0,002685 ; \\
& 0,018207 ; 0,003626 ; 0,004222 ; 0,004905 ; 0,013203 ; \\
& 0,008230 ; 0,000663 ; 0,001095 ; 0,002525 ; 0,001666 ; \\
& 0,007090 ; 0,016000 ; 0,020833 ; 0,014248 ; 0,001527 ; \\
& 0,002969 ; 0,002731 ; 0,003429 ;]
\end{aligned}
$$

A matriz inversa de uma matria diagonal é $\grave{t} a m-$ bém uma matriz diagonal e composta pelos inversos dos elementos da matriz original. Temos então

$$
\begin{array}{rl}
\mathrm{S}^{-1}=\operatorname{diag} . & {[356,633381 ; 166,666667 ; 34,133188 ; 261,164795 ;} \\
32 \times 32 & 1383,125864 ; 670,241287 ; 343,053173 ; 549,679487 ; \\
& 382,701875 ; 155,351872 ; 13,500014 ; 145,080023 ; \\
& 938,967136 ; 372,439479 ; 54 ; 923930 ; 275,785990 ; \\
& 236,854571 ; 203,873598 ; 75,740362 ; 121,506683 ; \\
1508,295626,913,242009 ; 396,039604 ; 600,240096 ; \\
141,043724 ; 62,500000 ; 48,000768 ; 10,185289 ; \\
654,878847 ; 336,813742 ; 366,166239 ; 291,630213 ;]
\end{array}
$$

3.3.3.6. Matriz $\mathrm{F}(\mathrm{p})$

$$
\text { A matriz } \underset{\sim}{F}(p) \text { é dada por } \underset{32 \times 64}{A} \cdot \underset{64 \times 1}{p} \cdot \text { Temos }
$$

então uma matriz coluna, que para simplificar, passamos a escrever em sua forma transposta. 


\begin{tabular}{|c|c|c|c|c|c|}
\hline \multirow{7}{*}[\underset{\sim}{\mathrm{F}}(\mathrm{p})]{$_{1 \times 32}^{\prime}$} & {$[4 / 64$} & $24 / 40$ & $5 / 8$ & $38 / 53$ & $182 / 231$ \\
\hline & $99 / 144$ & $17 / 18$ & $78 / 105$ & $55 / 92$ & $23 / 35$ \\
\hline & $2 / 3$ & $21 / 29$ & $139 / 212$ & $51 / 92$ & $5 / 13$ \\
\hline & $38 / 68$ & $35 / 51$ & $30 / 43$ & $11 / 18$ & $18 / 27$ \\
\hline & $.218 / 301$ & $135 / 197$ & $.58 / 82$ & $87 / 143$ & $19 / 35$ \\
\hline & $6 / 15$ & $6 / 12$ & $10 / 17$ & $92 / 160$ & $40 / 84$ \\
\hline & $50 / 65$ & $40 / 72$ & & & \\
\hline
\end{tabular}

\subsubsection{Matriz $\beta$}

A matriz $\beta$ è uma matriz coluna contendo os 32 parâmetros do modelo adotado. O que vamos determinar será a sua estimativa representada por $\stackrel{\mathrm{b}}{\tilde{3}}$ $\tilde{3} 2 \times 1$.

Conhecidas todas as matrizes, que são envolvidas, passamos a estimar os parâmetros através da fórmula,

$$
\begin{aligned}
& \hat{\beta}=b=\left[\begin{array}{lll}
X^{\prime} & S^{-1} & X
\end{array}\right]^{-1} \quad X^{\prime} \cdot S^{-1} F(p) \text { e chegamos a } \\
& b^{\prime}=\left[\begin{array}{llllll}
0,6395 & 0,0274 & 0,0641 & -0,0116 & -0,0086 & 0,0195
\end{array}\right. \\
& 1 \times 32 \\
& \begin{array}{llllll}
0,0028 & 0,0139 & 0,0050 & -0,0079 & -0,0208 & 0,0094
\end{array} \\
& \begin{array}{lllllll}
0,0084 & -0,0008 & -0,0230 & 0,0166 & -0,0384 & -0,2931
\end{array} \\
& \begin{array}{llllll}
0,0269 & -0,0096 & 0,0060 & 0,0060 & 0,0103 & 0,0041
\end{array} \\
& \begin{array}{llllll}
-0,0078 & 0,0166 & 0,0236 & -0,0074 & -0,0005 & -0,0115
\end{array} \\
& 0,0123 \quad 0,0274]
\end{aligned}
$$


3.3.3.8. A estimativa do modelo

$$
\begin{aligned}
\hat{Y} & =0,0395+0,0274 \mathrm{x}_{1}+0,0641 \mathrm{x}_{2}-0,0116 \mathrm{x}_{3}- \\
& -0,0086 \mathrm{x}_{4}+0,0195 \mathrm{x}_{5}+0,0028 \mathrm{x}_{1} \mathrm{x}_{2}+0,0139 \mathrm{x}_{1} \mathrm{x}_{3}+ \\
& +0,0050 \mathrm{x}_{1} \mathrm{x}_{4}-0,0079 \mathrm{x}_{1} \mathrm{x}_{5}-0,0208 \mathrm{x}_{2} \mathrm{x}_{3}+0,0094 \mathrm{x}_{2} \mathrm{x}_{4}+ \\
& +0,0084 \mathrm{x}_{2} \mathrm{x}_{5}-0,0008 \mathrm{x}_{3} \mathrm{x}_{4}-0,0230 \mathrm{x}_{3} \mathrm{x}_{5}+0,0166 \mathrm{x}_{4} \mathrm{x}_{5}+ \\
& -0,0384 \mathrm{x}_{1} \mathrm{x}_{2} \mathrm{x}_{3}-0,2931 \mathrm{x}_{1} \mathrm{x}_{2} \mathrm{x}_{4}+0,0269 \mathrm{x}_{1} \mathrm{x}_{2} \mathrm{x}_{5}- \\
& -0,0096 \mathrm{x}_{1} \mathrm{x}_{3} \mathrm{x}_{4}-0,0060 \mathrm{x}_{1} \mathrm{x}_{3} \mathrm{x}_{5}+0,0103 \mathrm{x}_{1} \mathrm{x}_{4} \mathrm{x}_{5}+ \\
& +0,0163 \mathrm{x}_{2} \mathrm{x}_{3} \mathrm{x}_{4}-0,0041 \mathrm{x}_{2} \mathrm{x}_{3} \mathrm{x}_{5}-0,0078 \mathrm{x}_{2} \mathrm{x}_{4} \mathrm{x}_{5}+ \\
& +0,0166 \mathrm{x}_{3} \mathrm{x}_{4} \mathrm{x}_{5}-0,0236 \mathrm{x}_{1} \mathrm{x}_{2} \mathrm{x}_{3} \mathrm{x}_{4}-0,0074 \mathrm{x}_{1} \mathrm{x}_{2} \mathrm{x}_{3} \mathrm{x}_{5}- \\
& -0,0005 \mathrm{x}_{1} \mathrm{x}_{2} \mathrm{x}_{4} \mathrm{x}_{5}-0,0115 \mathrm{x}_{1} \mathrm{x}_{3} \mathrm{x}_{4} \mathrm{x}_{5}+0,0123 \mathrm{x}_{2} \mathrm{x}_{3} \mathrm{x}_{4} \mathrm{x}_{5}+ \\
& +0,0274 \mathrm{x}_{1} \mathrm{x}_{2} \mathrm{x}_{3} \mathrm{x}_{4} \mathrm{x}_{5}
\end{aligned}
$$

3.3.3.9. Teste dos parâmetros encontrados

Testaremos as hipóteses Ho: $\beta_{i}=0$, onde $\beta_{i}$ re

presenta cada parâmetro de $\beta, \operatorname{com} i=1,2,3, \ldots, 32 \quad\left(\beta_{1}\right.$

não é testado pois representa a média do modelo) através do teste

$\mathrm{SQ}[\mathrm{C} \beta=0]=(\mathrm{Cb})^{\prime}\left[\mathrm{C}\left(\mathrm{X}^{\prime} \mathrm{S}^{-1} \mathrm{X}\right)^{-1} \mathrm{C}^{\prime}\right]^{-1}(\mathrm{Cb})=\mathrm{X}_{1}^{2}$

Para o efeito da idade (I) teremos

$\mathrm{H}_{01}: \alpha_{1}=0 \Rightarrow \mathrm{C}_{I} \beta=0 \mathrm{com}$

$\mathrm{C}_{\mathrm{I}}=\left[\begin{array}{llllllll}0 & 1 & 0 & 0 & 0 & . & . & 0\end{array}\right]$ 
Para o efeito do Tratamento

$$
\begin{aligned}
& \mathrm{Ho}_{2}: \alpha_{2}=0 \rightarrow \mathrm{C}_{\mathrm{T}^{\beta}}=0 \quad \text { com } \\
& \mathrm{C}_{\mathrm{T}}=\left[\begin{array}{lllllll}
0 & 0 & 1 & 0 & 0 & \ldots & 0
\end{array}\right] \\
& 1 \times 32 \\
& \text { Para o efeito do sexo }
\end{aligned}
$$

Para o efeito do Maxilar (M)

$\mathrm{Ho}_{4}: \alpha_{4}=0 \rightarrow \mathrm{C}_{M^{\beta}}=0$ com

$\mathrm{C}_{\mathrm{M}}=\left[\begin{array}{llllllll}0 & 0 & 0 & 0 & 1 & 0 & \ldots & 0\end{array}\right]$

$1 \times 32$

Para o efeito do número de raízes (R)

$\mathrm{Ho}_{5}: \alpha_{5}=0 \rightarrow \mathrm{C}_{\mathrm{R}} \beta=0$ com

$\mathrm{C}_{\mathrm{R}}=\left[\begin{array}{lllllllll}0 & 0 & 0 & 0 & 0 & 1 & 0 & \ldots & 0\end{array}\right]$

$1 \times 32$

Como podemos notar, para cada Hoi temos como varıação, somente a posição da unidade (1) dentro da matriz C. Fazendo i variar da 2 a a a é a 32 a coluna de $C$, testaremos todos os parâmetros do modelo adotado exceto a média ( $\mu$ ). 
Estes testes fornecem os seguintes resultados:

\begin{tabular}{|c|c|c|c|}
\hline FONTE DE VARIAÇÃO & G. L. & $\mathrm{X}^{2}$ OBTIDO & SIGNIFICÂNCIA \\
\hline IDADE (I) & 1 & 2,8407 & s. \\
\hline TRATAMENTO (T) & 1 & 15,5903 & s. \\
\hline SEXO (S) & 1 & 0,5106 & $\mathrm{n} \cdot \mathrm{s}$. \\
\hline MAXILAR (M) . & 1 & 0,2834 & n.s. \\
\hline$N^{\circ}$ DE RAIZES (R) & 1 & 1,4430 & s. \\
\hline $\mathrm{IXT}$ & 1 & 0,0308 & $\mathrm{n} \cdot \mathrm{s}$. \\
\hline IXS & 1 & 0,7361 & $\mathrm{n} \cdot \mathrm{s}$ \\
\hline IXM & 1 & 0,0966 & $\mathrm{n} \cdot \mathrm{s} \cdot$ \\
\hline IXR & 1 & 0,2356 & $\mathrm{n} \cdot \mathrm{s} \cdot$ \\
\hline TXS & 1 & 1,6441 & s. \\
\hline $\mathrm{TXM}$ & 1 & 0,3335 & $\mathrm{n} \cdot \mathrm{s}$ \\
\hline $\mathrm{TXR}$ & 1 & 0,2684 & $\mathrm{n} \cdot \mathrm{s}$ \\
\hline SXM & 1 & 0,0026 & $\mathrm{n} \cdot \mathrm{s}$. \\
\hline SXR & 1 & 2,0019 & s. \\
\hline MXR & 1 & 1,0431 & s. \\
\hline IXTXS & 1 & 5,5960 & s. \\
\hline IXTXM & 1 & 3,2608 & s. \\
\hline IXTXR & 1 & 2,7505 & $\mathrm{~s}$. \\
\hline IXSXM & 1 & 0,3502 & n.s. \\
\hline IXSXR & 1 & 0,1345 & $\mathrm{n} \cdot \mathrm{s}$. \\
\hline I XMXR & 1 & 0,4028 & $\mathrm{n} \cdot \mathrm{s}$. \\
\hline TXSXM & 1 & 1,0103 & s. \\
\hline TXSXR & 1 & 0,0640 & $\mathrm{n} \cdot \mathrm{s}$. \\
\hline TXMXR & 1 & 0,2303 & $\mathrm{n} \cdot \mathrm{s}$. \\
\hline SXMXR & 1 & 1,0470 & $\mathrm{~s}$ \\
\hline IXTXSXM & 1 & 2,1062 & $\mathrm{~s}$. \\
\hline IXTXSXR & 1 & 0,2089 & $\mathrm{n} \cdot \mathrm{s}$. \\
\hline IXTXMXR & 1 & 0,0008 & $\mathrm{n} \cdot \mathrm{s}$. \\
\hline I XSXMXR & 1 & 0,5036 & $\mathrm{n} \cdot \mathrm{s}$. \\
\hline TXSXMXR & 1 & 0,5763 & $\mathrm{n} \cdot \mathrm{s}$. \\
\hline IXTXSXMXR & 1 & 2,8573 & $\mathrm{~s}$. \\
\hline TOTAL & 31 & & \\
\hline
\end{tabular}

n.s. = não significativo

s. = significativo (considerado significativo os valores encontrados > 1) 


\subsubsection{Readaptando o modelo}

Desprezando os efeitos não significativos (con sideramos significativos valores obtidos maiores que um), pas samos a trabalhar com um novo modelo matemático que é reduzido em relação ao adotado anteriormente.

Temos então:

$$
\begin{aligned}
Y^{\prime}{ }_{i} & =\mu^{\prime} \quad \alpha^{\prime}{ }_{i} \mathrm{x}+\alpha^{\prime}{ }_{2} \mathrm{x}_{2}+\alpha^{\prime}{ }_{5} \mathrm{x}_{5}+\beta^{\prime}{ }_{5} \mathrm{x}_{2} \mathrm{x}_{3}+\beta^{\prime}{ }_{9} \mathrm{x}_{3} \mathrm{x}_{5}+ \\
& +\beta^{\prime}{ }_{10} \mathrm{x}_{4} \mathrm{x}_{5}+\gamma^{\prime}{ }_{1} \mathrm{x}_{1} \mathrm{x}_{2} \mathrm{x}_{3}+\gamma^{\prime}{ }_{2} \mathrm{x}_{1} \mathrm{x}_{2} \mathrm{x}_{4}+\gamma^{\prime}{ }_{3} \mathrm{x}_{1} \mathrm{x}_{2} \mathrm{x}_{5}+ \\
& +\gamma^{\prime}{ }_{7} \mathrm{x}_{2} \mathrm{x}_{3} \mathrm{x}_{4}+\gamma^{\prime}{ }_{10} \mathrm{x}_{3} \mathrm{x}_{4} \mathrm{x}_{5}+{ }^{\prime}{ }_{1} \mathrm{x}_{1} \mathrm{x}_{2} \mathrm{x}_{3} \mathrm{x}_{4}+{ }^{\prime}{ }_{1} \mathrm{x}_{1} \mathrm{x}_{2} \mathrm{x}_{3} \mathrm{x}_{4} \mathrm{x}_{5} \\
& +l_{i}
\end{aligned}
$$

A partir deste novo modelo formamos uma nova matriz $X$ de planejamento, que para diferenciar chamaremos de $x_{1}$ e uma nova $\beta$ que chamaremos $\beta_{1}$.

\subsubsection{Matriz $x_{1}$}

Para a formação da matriz $x_{1}$, eliminamos da ma triz $X$ as colunas relativas aos parâmetros desprezados e temos uma matriz reduzida no seu número de colunas. Assim $x_{1}$ te rā o mesmo número de linhas (32) e o número de colunas passa de 32 para 14 que é o número de parâmetros do modelo reduzido.

\subsubsection{Matriz $\beta_{1}$}

A matriz $\beta_{1}$ é formada pelos 14 parâmetros do 
novo modelo e a matriz $b_{1}$ pelas estimativas destes parâmetros. As demais matrizes permanecem iguais, pois independem do modelo adotado.

3.3.4:3. Estimando o novo modelo

A estimativa é dada por

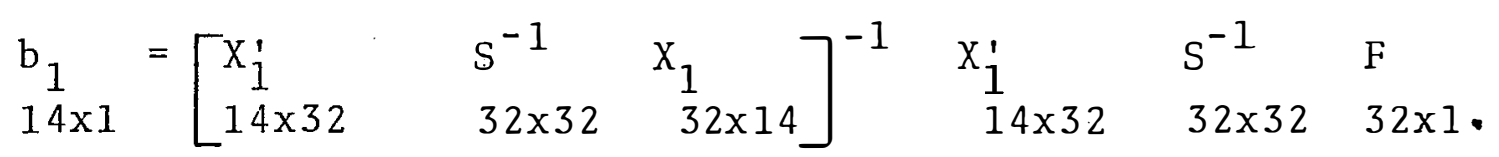

A matriz das estimativas obtidas $\left(b_{1}\right)$ é apresentada a seguir em sua forma transposta.

$\mathrm{b}_{i}=\left[\begin{array}{lllllll}0,6490 & 0,0270 & 0,0637 & 0,0216 & -0,0147 & -0,0285\end{array}\right.$ $\begin{array}{lllllll}0,0084 & -0,0323 & -0,0235 & 0,0271 & 0,0070 & 0,0198\end{array}$ $0,0213[0,0179]$

Portanto a estimativa do modelo reduzido fica:

$$
\begin{aligned}
\hat{Y}_{i}^{\prime=} & 0,0490 x_{0}+0,0270 x_{1}+0,0637 x_{2}+0,0216 x_{5}- \\
& -0,0147 x_{2} x_{3}-0,0285 x_{3} x_{5}+0,0084 x_{4} x_{5}-0,0323 x_{1} x_{2} x_{3} \\
& -0,0235 x_{1} x_{2} x_{4}+0,0271 x_{1} x_{2} x_{5}+0,0070 x_{2} x_{3} x_{4}+ \\
& 0,0198 x_{3} x_{4} x_{5}+0,0213 x_{1} x_{2} x_{3} x_{4}+0,0179 x_{1} x_{2} x_{3} x_{4} x_{5}
\end{aligned}
$$

3.3.4.4. As proporções obtidas e as estimadas através modelo reduzido

Para ilustração apresentamos uma comparação das proporções estimadas e obtidas através do modelo acima(Y $\hat{Y}_{i}$ 
representa a estimativa da mesma proporção).

$$
\begin{array}{lllll}
Y_{1}=0,7656 & \hat{Y}_{1}=0,7368 & Y_{17}=0,6863 & \hat{Y}_{17}=0,6888 \\
Y_{2}=0,6000 & \hat{Y}_{2}=0,6597 & Y_{18}=0,6977 & \hat{Y}_{18}=0,7362 \\
Y_{3}=0,6250 & \hat{Y}_{3}=0,6620 & Y_{19}=0,6111 & \hat{Y}_{19}=0,6498 \\
Y_{4}=0,7170 & \hat{Y}_{4}=0,7138 & Y_{20}=0,6667 & \hat{Y}_{20}=0,7384 \\
Y_{5}=0,7879 & \hat{Y}_{5}=0,7828 & Y_{21}=0,7243 & \hat{Y}_{21}=0,7354 \\
Y_{6}=0,6875 & \hat{Y}_{6}=0,6870 & Y_{22}=0,6853 & \hat{Y}_{22}=0,6764 \\
Y_{7}=0,9444 & \hat{Y}_{7}=0,9450 & Y_{23}=0,7073 & \hat{Y}_{23}=0,6468 \\
Y_{8}=0,7429 & \hat{Y}_{8}=0,7320 & Y_{24}=0,6084 & \hat{Y}_{24}=0,6138 \\
Y_{9}=0,5978 & \hat{Y}_{9}=0,6308 & Y_{25}=0,5429 & \hat{Y}_{25}=0,5978 \\
Y_{10}=0,6571 & \hat{Y}_{10}=0,6782 & Y_{26}=0,4000 & \hat{Y}_{26}=0,4652 \\
Y_{11}=0,6667 & \hat{Y}_{11}=0,6198 & Y_{27}=0,5000 & \hat{Y}_{27}=0,5240 \\
Y_{12}=0,7241 & \hat{Y}_{12}=0,7084 & Y_{28}=0,5882 & \hat{Y}_{28}=0,5758 \\
Y_{13}=0,6557 & \hat{Y}_{13}=0,6466 & Y_{29}=0,5750 & \hat{Y}_{29}=0,5860 \\
Y_{14}=0,5543 & \hat{Y}_{14}=0,5876 & Y_{30}=0,4762 & \hat{Y}_{30}=0,4902 \\
Y_{15}=0,3846 & \hat{Y}_{15}=0,5300 & Y_{31}=0,7692 & \hat{Y}_{31}=0,7274 \\
Y_{16}=0,5588 & \hat{Y}_{16}=0,4970 & Y_{32}=0,5556 & \hat{Y}_{32}=0,5072
\end{array}
$$

Novamente testamos as Hipóteses Ho: $\beta_{i}=0$, p $\underline{a}$ ra $i=1,2,3, \ldots, 14$, através do teste

$S Q[C \beta=0]=\left(C b_{1}\right) \cdot\left[C\left(x_{1}^{\prime} s^{-1} x_{1}\right)^{-1} C^{\prime}\right]^{-1} \mathrm{Cb}_{1}=x_{1}^{2}$

Testamos também o ajustamento do modelo pois só agora temos disponibilidade de graus de liberdade. Anteriormente tinhamos 31 graus de liberdade para testarmos os 31 parâmetros, agora temos apenas 13 parâme 
tros a serem testados o que nos dā uma diferença de 18 graus de liberdade para o teste do ajustamento do modelo que é feito através do teste

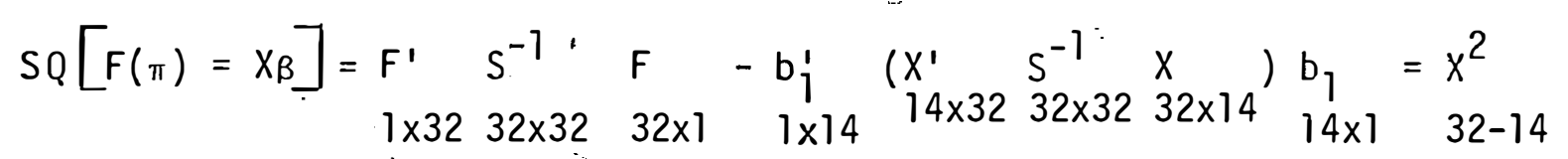
Aplicando os testes aos parâmetros e ao modelo chegamos ao seguinte quadro:

\begin{tabular}{|c|c|c|c|}
\hline FONTE DE VARIAÇÃO & G. L. & $x^{2}$ & SI GNI FI CÂNČ IA \\
\hline IDADE (I) & 1 & 8,1754 & * * \\
\hline TRATAMENTO (T) & 1 & 29,4918 & ** \\
\hline NUMERO DE RAIZES (R) & 1 & 3,0880 & n.s. \\
\hline TXS & 1 & 1,4651 . & $\mathrm{n} \cdot \mathrm{s}$. \\
\hline SXR & 1 & 5,3137 & $\mathrm{n} \cdot \mathrm{s}$. \\
\hline MXR & 1 & 0,4241 & $\mathrm{n} \cdot \mathrm{s}$. \\
\hline IXTXS & 1 & 9,1905 & ** \\
\hline IXTXM & 1 & 3,8411 & $\mathrm{n} \cdot \mathrm{s}$. \\
\hline IXTXR & 1 & 7,4624 & ** \\
\hline TXSXM & 1 & 0,4871 & $\mathrm{n} \cdot \mathrm{s} \cdot$ \\
\hline SXMXR & 1 & 2,5717 & $\mathrm{n} \cdot \mathrm{s} \cdot$ \\
\hline IXTXSXM & 1 & 3,1408 & $\mathrm{n} \cdot \mathrm{s} \cdot$ \\
\hline IXTXSXMXR & 1 & 3,2371 & $\mathrm{n} \cdot \mathrm{s}$. \\
\hline AJUSTE OU ERRO & 18 & $8,77.54$ & $\mathrm{n} \cdot \mathrm{s} \cdot$ \\
\hline TOTAL & 31 & & \\
\hline
\end{tabular}

onde

n.s. não significante

* * significante a $1 \%$ 
4. DISCUSSÃO

1ạ O fator Idade foi significativo a $1 \frac{\circ}{0}$, isto é, dos dois níveis considerados um influiu mais que o outro na reatividade. Podemos notar na Tabela 5 que o nível "menos que trinta anos" é responsável por maior reatividade.

TABELA 5: Frequência e proporçōes da reatividade relativas à IDADE

\begin{tabular}{lcccc}
\hline IDADE & COM REAÇÃ & SEM REAÇAO & TOTAL & $\mathrm{p}$ \\
\hline$<30$ anos & 826 & 381 & 1207 & 0,6843 \\
$\geqq 30$ anos & 855 & 467 & 1322 & 0,6467 \\
\hline TOTAIS & 1681 & 848 & 2529 & \\
\hline
\end{tabular}


2. O fator Tratamento foi significativo a 1\%,o que quer dizer que estatisticamente os dois tratamentos diferem-se. Na tabela 6 podemos notar que o tratamento parcial é responsāvel por maior incidência de reatividade.

TABELA 6: Frequência e proporções da reatividade relativas ao Tratamento

\begin{tabular}{lcccc}
\hline TRATAMENTO & COM REAÇÃO & SEM REAÇÃO & TOTAL & $\mathrm{p}$ \\
\hline PARCIAL & 1084 & 441 & 1525 & 0,7108 \\
TOTAL & 597 & 407 & 1004 & 0,5946 \\
\hline TOTAIS & 1681 & 848 & 2529 & \\
\hline
\end{tabular}

3. A interação Idade X Tratamento foi não significativa quando a testamos pelo modelo completo, o que pode ser observado na tabela 7 e 8 , onde o fator Idade não altera a variabilidade exercida pelo fator Tratamento sobre a reatividade e vice e versa. Porém, esta interação altera-se significativamente na presença dos fatores principais Sexo e Número de Raízes.

Essas alterações podem ser notadas nas tabelas $9,10,11$ e 12 . 
TABELA 7: Interação Idade $x$ Tratamento (frequência)

\begin{tabular}{|c|c|c|c|c|c|c|}
\hline TRATAMENTO & $\mathrm{P} A \mathrm{~A}$ & C I A L & & $0 \mathrm{~T}$ & $\mathrm{~L}$ & \\
\hline IDADE & $<30$ & $\geqq 30$ & & & $\geqq 3$ & \\
\hline REATIVIDADE & C & $\cdots \mathrm{C}$ & $\mathrm{C}$ & S & $\mathrm{C}$ & s \\
\hline FREQUENCI A & $4.92 \quad 171$ & $592 \quad 270$ & 334 & 210 & 263 & 197 \\
\hline
\end{tabular}

TABELA 8: Interação Idade $x$ Tratamento (proporções)

\begin{tabular}{l|ccccc}
\hline TRATAMENTO & \multicolumn{2}{|c}{ PARCIAL } & & \multicolumn{2}{c}{ TOTAL } \\
\cline { 2 - 3 } \cline { 5 - 6 } IDADE & $<30$ & & & $<30$ & $\geqq 30$ \\
\hline PROPORÇAO & 0,7421 & 0,6868 & & 0,6140 & 0,5717 \\
\hline
\end{tabular}

Notamos nessa tabela que $\mathrm{p}$ apresenta o mesmo decréscimo entre os níveis do fator Tratamento dentro de cada nivel do fator Idade e entre os niveis do fator Idade dentro de 'cada nível do fator Tratamento

TABELA 9: Interação Idade $x$ Tratamento $x$ Sexo (frequência)

\begin{tabular}{|c|c|c|c|c|c|c|c|c|c|}
\hline \multirow{3}{*}{\multicolumn{2}{|c|}{$\begin{array}{l}\text { TRAT AMENTO } \\
\text { IDADE } \\
\text { REAT IVIDADE }\end{array}$}} & \multicolumn{4}{|c|}{$P A R \quad C \quad I A L$} & \multicolumn{4}{|c|}{$\mathrm{T} O \mathrm{O} \quad \mathrm{T} A \mathrm{~L}$} \\
\hline & & \multicolumn{2}{|c|}{$<30$} & \multicolumn{2}{|c|}{$\geqq 30$} & \multicolumn{2}{|c|}{$<30$} & \multicolumn{2}{|c|}{$\geqq 30$} \\
\hline & & $\mathrm{C}$ & $S$ & $\mathrm{C}$ & $S$ & $\mathrm{C}$ & $S$ & $\mathrm{C}$ & $\mathrm{S}$ \\
\hline 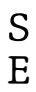 & MASCULINO & 116 & 49 & 94 & 45 & 101 & 58 & 41 & 38 \\
\hline X & FEMI N I NO & 376 & 122 & 498 & 225 & 233 & 152 & 222 & 159 \\
\hline
\end{tabular}


TABELA 10: Interação Idade $x$ Tratamento $x$ Sexo (proporções)

\begin{tabular}{|c|c|c|c|c|c|}
\hline \multirow{2}{*}{\multicolumn{2}{|c|}{$\begin{array}{l}\text { TRAT AMENTO } \\
\text { IDADE }\end{array}$}} & \multicolumn{2}{|c|}{ PARCIAL } & \multicolumn{2}{|c|}{ TOTAL } \\
\hline & & \multirow{2}{*}{$\frac{<30}{0,7030}$} & \multirow{2}{*}{$\frac{\geqq 30}{0,6763}$} & \multirow{2}{*}{$\frac{<30}{0,6352}$} & \multirow{2}{*}{$\frac{\geqq 30}{0,5190}$} \\
\hline $\begin{array}{l}\mathrm{S} \\
\mathrm{E}\end{array}$ & MASCULINO & & & & \\
\hline$\hat{0}$ & FEMININO & 0,7550 & 0,6888 & 0,6052 & 0,5827 \\
\hline
\end{tabular}

Notamos que, o sexo feminino apresentou a proporção. p maior em todos os níveis da interação Tratamento $x$ Idade, com excessão do tratamento total na idade $<30$.

TABELA 11: Interação Idade $x$ Tratamento $x$ nọ de Raízes (frequência)

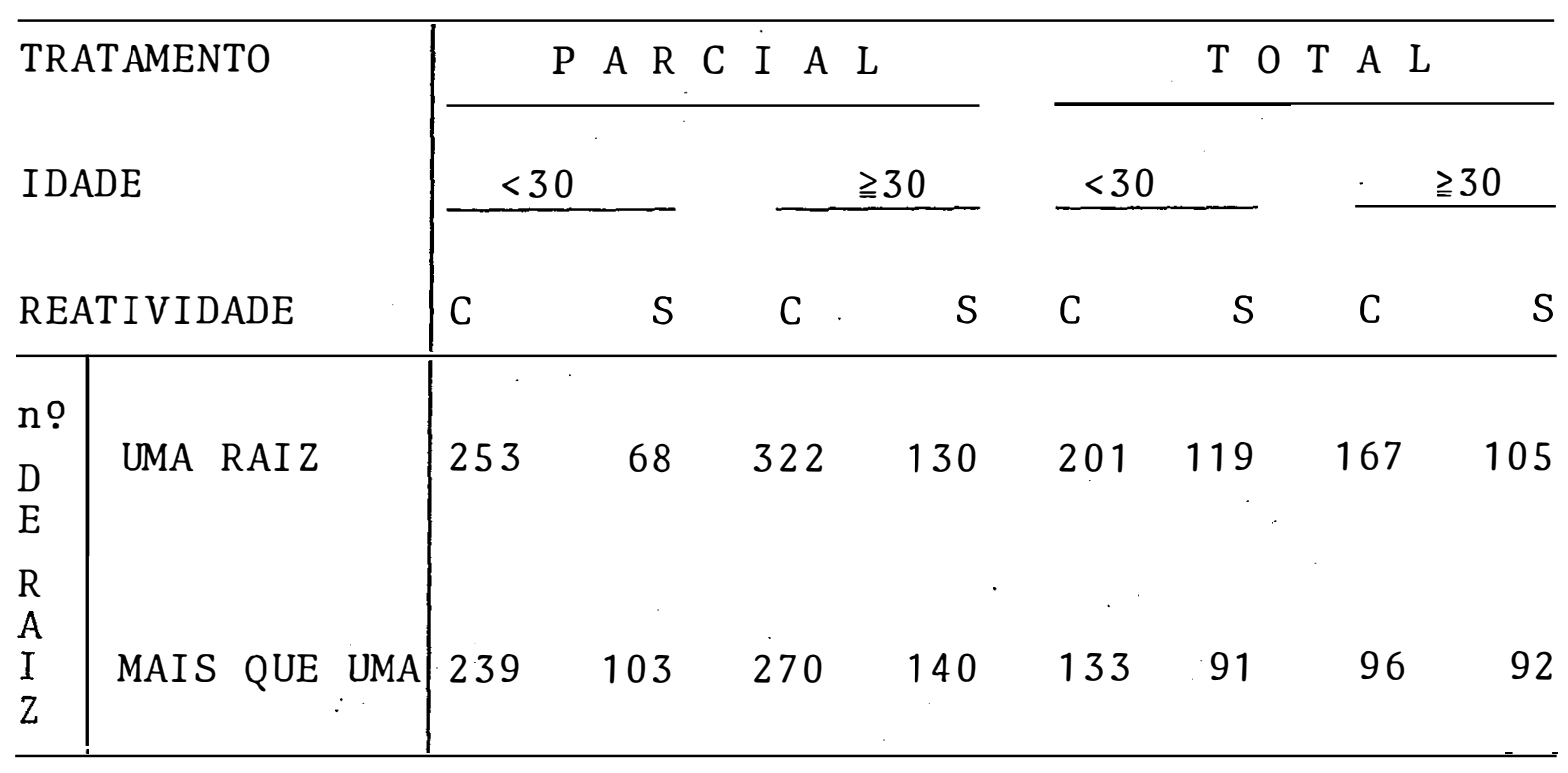


TABELA 12: Interação Idade $x$ Tratamento $x$ no de Raízes (proporções )

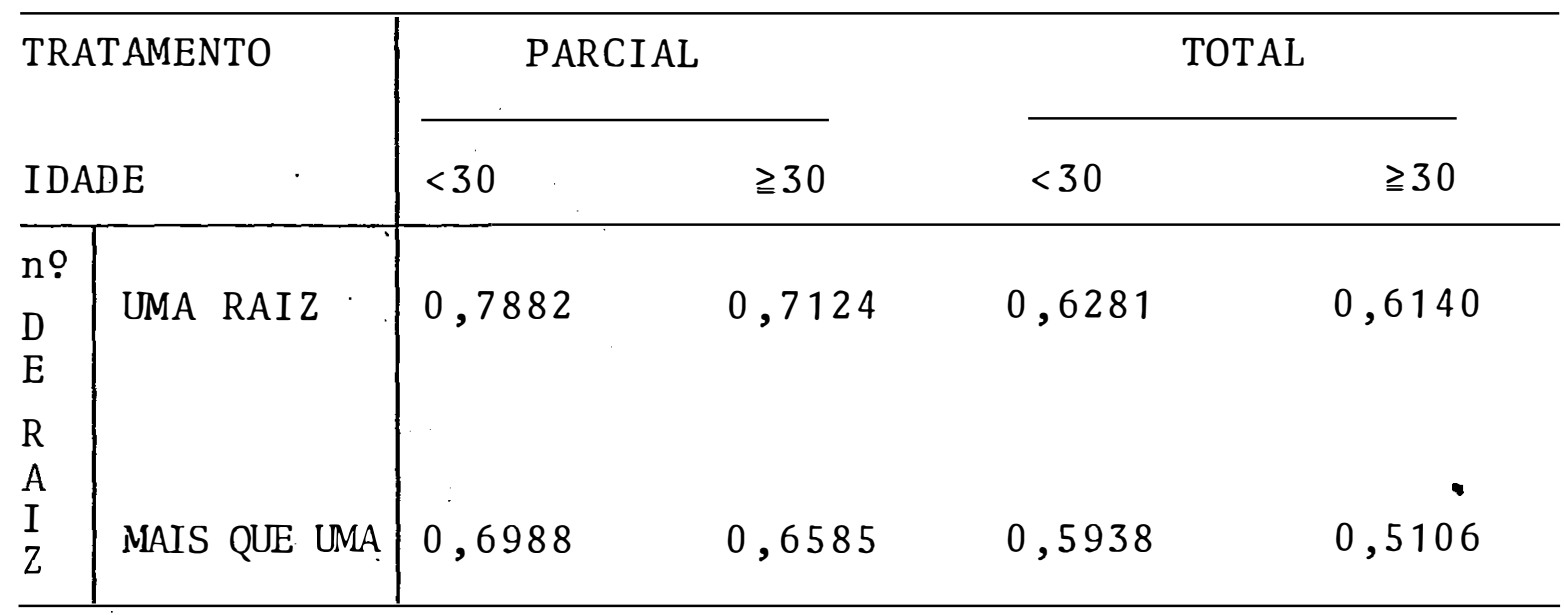

Notamos que, p decresce entre os dentes com apenas uma raiz e os dentes com mais que uma raiz em todos os níveis da interação Tratamento $x$ Idade, mas de modo bastante diferenciado, o que explica a significância da interação Tratamento $x$ Idade $x$ Nümero de Raízes.

4. A não significância do ajuste mostra que o modelo è adequado para explicar o experimento. 
5. CONCLUSOES FINAIS

Finalizando este trabalho faremos alguns comen tários sobre o método de análise de dados categorizados por modelos lineares proposto por GRIZZLE, STARMER e KOCH e as mo dificações a ele propostas por diversos autores.

O método GSK tem vantagens sobre os outros tes tes estatísticos usuais, essas vantagens estão: na não necessidade de estimadores de mäxima verossimilhança dos parâmetros sob $H_{0}$, que em algumas hipóteses apresenta dificuldades na obtenção desses estimadores; na diversificação dos delinea mentos possíveis de serem analisados e na oportunidade de determinarmos curvas ou superfícies de respostas, possibilitando uma análise detalhada de cada um dos parâmetros envolvidos, sejam eles, relativos a fatores principais ou às suas 
interações.

Apesar de não ter sido esta nossa preocupação constatamos através da literatura que o método e suas modificações permitem diversos tipos de estimação de parâmetros através de funções adequadas, que podem ser funções lineares, logarítmicas, exponenciais e algumas funções compostas.

Diversos autores criticam o método apontando muitas dificuldades de cảlculo, porém, da maneira que expusemos a metodologia do GSK e com a crescente utilização do "micro computador nas anālises estatísticas essas dificuldades apontadas desaparecem com uma programação bem simplificada en volvendo produtos e inversões de matrizes. Os programas de computação podem ser construídos a partir de combinações de programas já prontos e bem conhecidos, de produtos e inversões de matrizes, restando ao analista a construção das matri zes envolvidas. Salientamos ainda que essa construção pode também ser feita através de programação do computador.

$\mathrm{Na}$ anālise do experimento odontológico apresen tado neste trabalho, encontramos uma superfície de resposta capaz de explicā-1o de maneira aceitāvel, não só pela não rejeição do modelo como também pela comparação entre as proporções obtidas e as estimada através do modelo reduzido.

Vārios métodos para anālise de dados categorizados existem, o GSK foi o método estudado e por tudo que expusemos neste trabalho estamos convencidos de que se trata de um bom teste para grandes amostras e que para os casos de pequenas amostras seus resultados merecem ainda um estudo maior. 
6. B IBLIOGRAFIA

BERKSON, J., 1955. Maximum likelihood and minimum $X^{2}$ estimate of the logistic function. JASA. Boston, 50: 130-162.

BERKSON, J., 1968. Application of minimum logit $X^{2}$ estimate to a problem of Grizzle, with a natation on the problem of no interaction. Biometrics. Raleigh, 24: 75-95.

BHAPKAR, V.P., 1961. "Some test for categorical data", in: ANN. MATH. STAT., 32: 72-83.

BHAPKAR, V.P., 1966. A note on the equivalence of two test criteria for hupotheses in categorical data. JASA. Boston, 61: $228-235$.

BHAPKAR, V.P. e G.G. KOCH, 1968b. On the hypotheses of 'no interaction' in contingency tables. Biometrics. Raleigh, 24: 567-594. 
GOODMAN, L.A., 1968. The analysis of cross-classified data; independence, quasi-independence, and interaction in contingency tables with or without missing entries. JASA. Boston, 63: 1091-131.

GOODMAN, L.A., 1971b. The analysis ou multidimensional contingency tables: stepwise procedures and direct estimation methods for building models for multiple classifications. Technometrics. Washington, 13: 33-63.

GRIZZLE, J.E., C.F. STARMER e C.G. KOCH, 1969. Analysis of categorical data by linear models. Biometrics. Ral"eigh, 25: $489-504$.

GRIZZLE, J.E. e O.D. WILLIAMS, 1972. Log Linear models and tests of independence for contingency tables. Biometrics. Raleigh, 28 : 137-156.

JOHNSON, W.D. e G.G. KOCH, 1971. A note on the weighted least squares analysis of the Ries-Smith contingency table data. Technometrics. Washington, 13: 438-447.

LANDIS, J.R.; W.M. STANISH; J.L. FREEMAN e G.G. KOCH, 1976. A computer program for the generalized chi-square analysis of categorical data using weighted least squares (GENCAT). Computer Programs in Biomedicine, 6: 196-231.

NEYMAN, J., 1949. Contributions to the theory of the $\mathrm{X}^{2}$ test. Proc. Berkley Symp. Math. Statist. Prob. University of California Press. 239-272.

OGLIARI, P.J. 1984. Análise de Dados Categorizados: Modelo Log-Linear e Independência em tabelas de Contingência. USP Piracicaba (Dissertação de Mestrado). 
WADA, C.Y., 1977. Anālise de dados categóricos. Instituto de Matemática e Estatística - USP. (Dissertação de Mestrado).

WALD, A., 1943. Tests of statistical hypotheses concerning se veral parameters when the number of observations is large, In: TRANS. AMER. MATH. SOC., 54, 426-482. 
7. BIBLIOGRAFIA COMPLEMENTAR

BHAPKAR, V.P. e G.G. KOCH, 1968a. Hypotheses of 'no interaction' in multidimensional contingency tables. Technometrics. Washington, 10: 107-123.

BISHOP, Y.M.M., 1972. Effects Collapsing Multidimensional Contingency Tables. Biometrics, 24: 567-594.

CANTON, A.W.P., 1980. Anālise de dados categorizados. Atas do

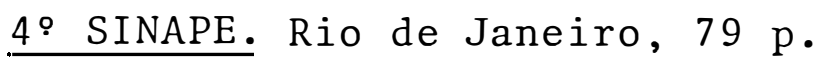

CANTON, A.W.P. e M.N. FESTA, 1979. Anālise de dados categorizados usando modelos lineares e suas aplicações - uma revisão bibliográfica. Atas do $3{ }^{\circ}$ SINAPE. São Paulo, 182-189.

CHI, G.Y.H.; G.G. KOCH; S. LUI CHI; W.M. STANISH; W.D. JOHNSON e J.R. LANDIS, 1978. A computer program for the generalized chi-square analysis of computing risks agrouped survival categorical data (CRISCAT). Computer programs in Biomedici nal, impress. 
DARROCH, J.N., 1962. Interactions in multi-factor contingency tables; In: J. ROYAL STAT. SOC., Series B, 24, 251-263.

FORTHOFER, R.N. e G.G. KOCH, 1973. An analysis for compounded functions of categorical data; In: BIOMETRICS, 29, 143-157.

GOODMAN, L.A., 1970. The multivariate analysis of qualitative data: interaction among multiple classifications. JASA. Boston, 65: 226-256.

GOODMAN, I.A., 1971a. Partitioning of chi-square analysis of marginal contingency tables, and estimation of experted frequencies in multidimensional contingency tables. JASA. Boston, 66: 339-344.

KOCH, G.G. e D.W. REINFURT, 1971. The analysis of categorical data from mixed models, In: BIOMETRICS, 27, 157-174.

KOCH, G.G., P.B. IMREY e D.W. REINFURT; 1972. Linear models analysis, of categorical data with imcomplete response vectors, In: BIOMETRICS, 663-690.

KOCH, G.G., W.D. JOHNSON e H.D. TOLLEY, 1972. A linear models approach to analysis of survivaland extent of disease in multidimensional contingency tables, In: JASA, 67, 780-795.

KOGH, G.G.; J.R. LANDIS; D.H. FREEMAN Jr.; J.L. FREEMAN e R. G. LEHNEN, 1978. A general methodology for the analysis of experiments with repeated measurement of categorical data. Biometrics. Raleigh, 33: 133-134.

KULLBACK, S.; H.H. KU e R. VARNER, 1971. Analysis of multidimensional contingency tables. JASA. Boston, 66: 55-64. 
MANTEL, N., 1963. Chi-square tests with ond degree of freedom; extensions of the Mantel-haenzel procedure, In: JASA, 58 , 690-700.

ODOROFF, C.L., 1970. Minimum logit chi-square estimation and maximum likelihood estimation in contingency tables. JASA. Boston, 65: 1617-32.

ROY, S.N. e M.A. KASTENBRAUM, 1956. On the hypotheses of no interaction in a multi-way contingency tables. Ann. Math. Statist. Baltimore, 27: 749-757.

WILLIAMS, O.D. e J.E. GRIZZLE, 1972. Analysis of contingency tables having ordered response categories, In: JASA, 67 , 55-63. 
$-69-$

A P E N D I C E 


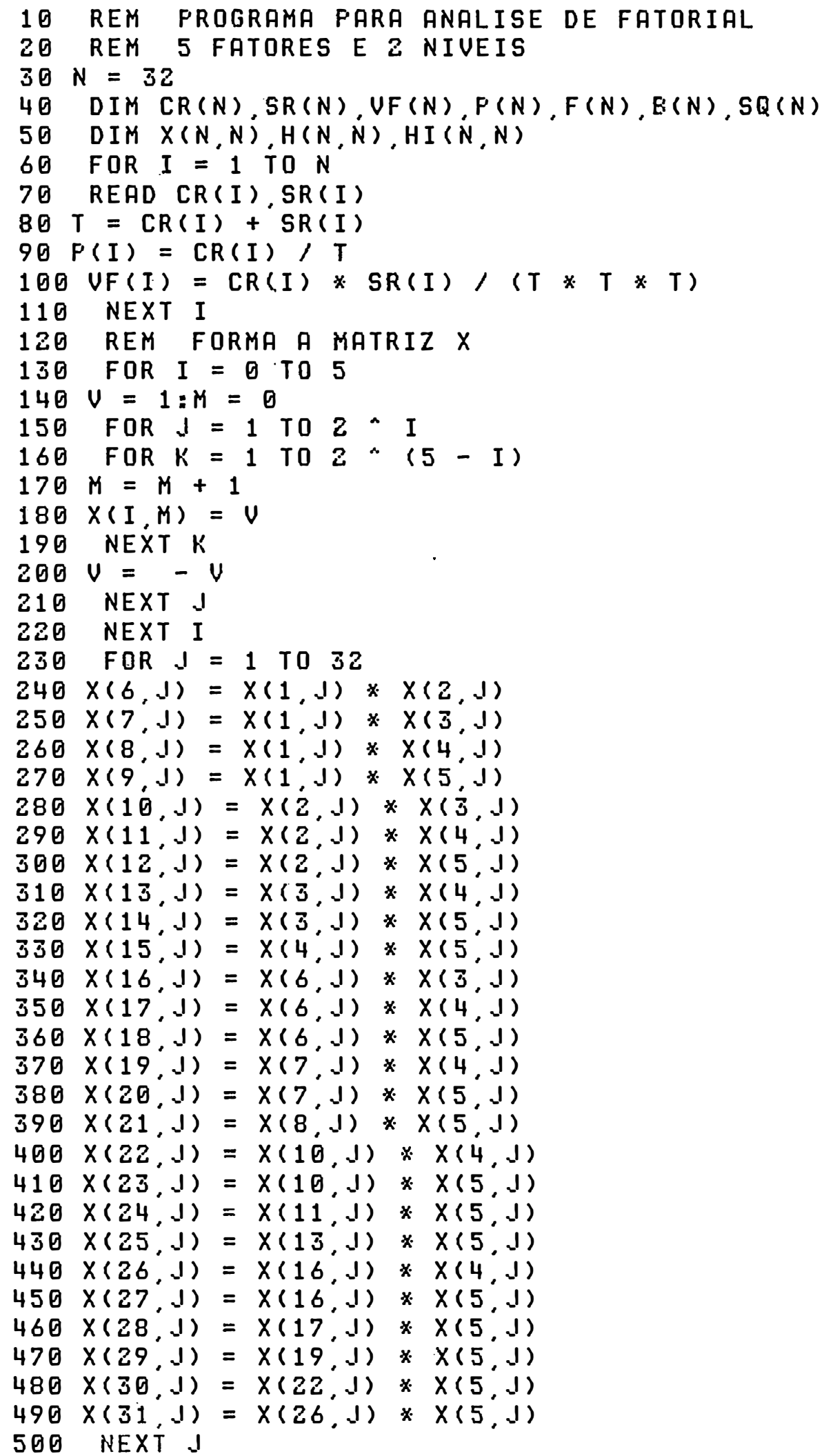




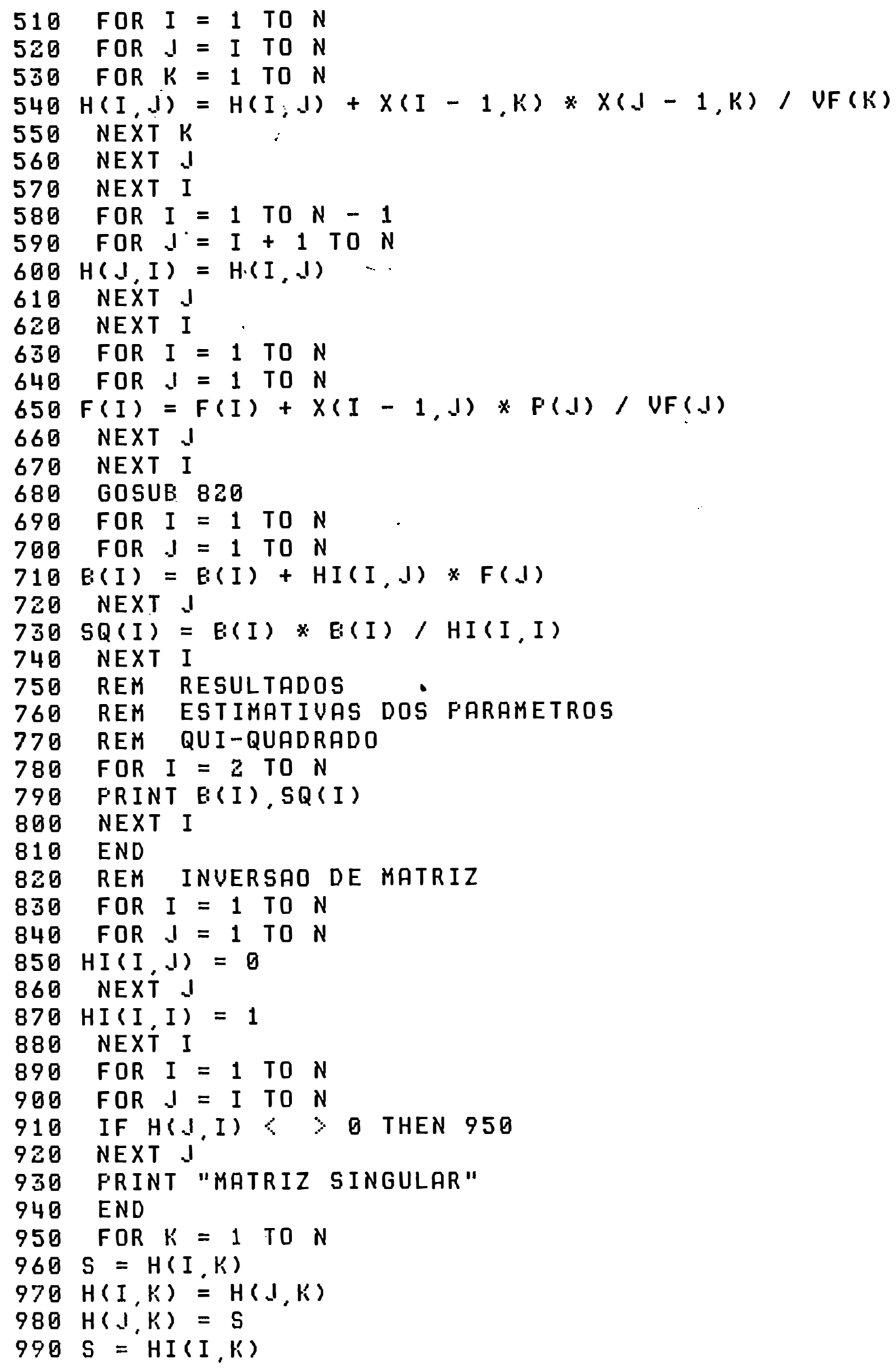




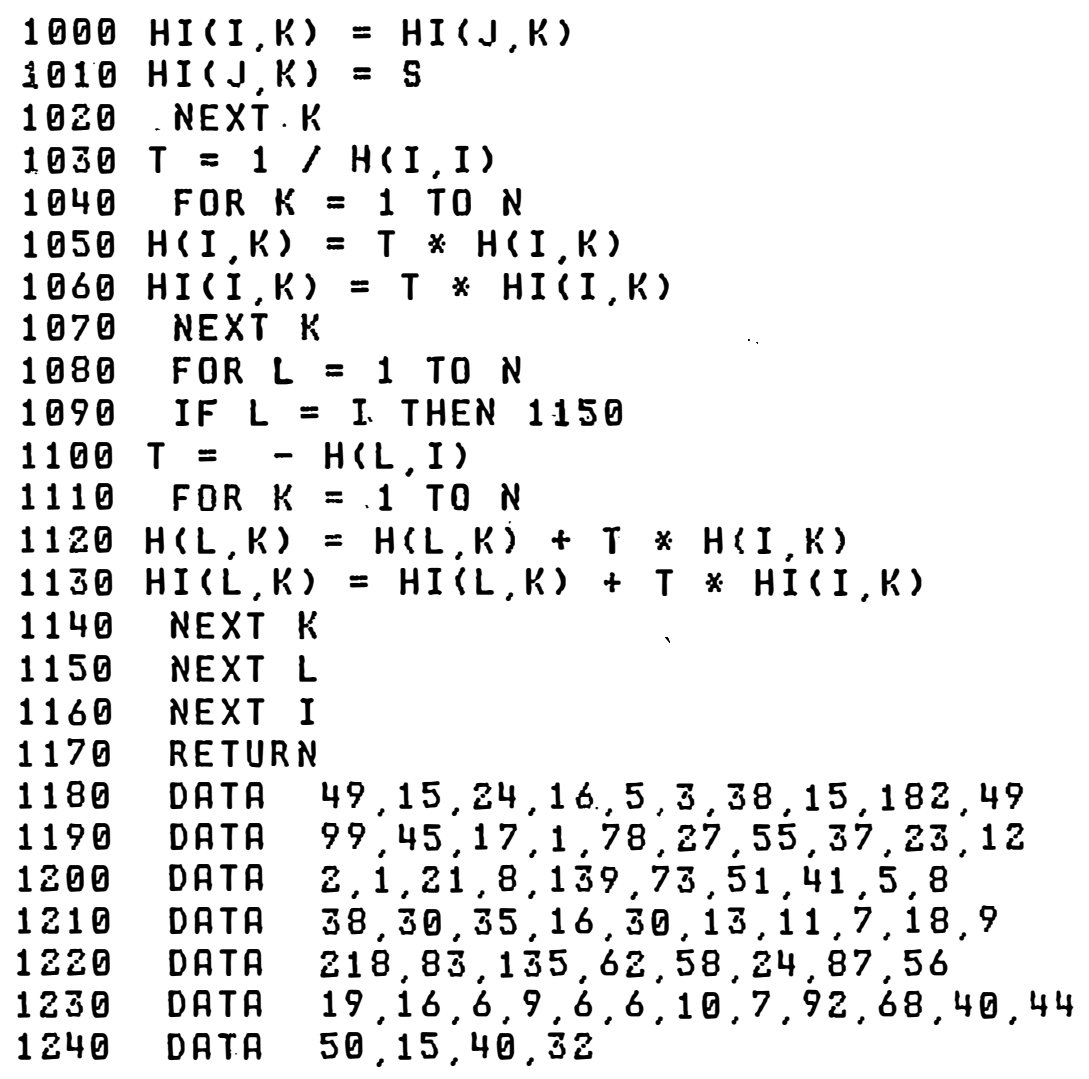

